\title{
On a system of Riemann-Liouville fractional differential equations with coupled nonlocal boundary conditions
}

Rodica Luca ${ }^{1 *}$ (1)

*Correspondence:

rlucatudor@yahoo.com

1 Department of Mathematics, Gh.

Asachi Technical University, 11 Blvd.

Carol I, nr. 11, lasi, 700506, Romania

\begin{abstract}
We investigate the existence of solutions for a system of Riemann-Liouville fractional differential equations with nonlinearities dependent on fractional integrals, subject to coupled nonlocal boundary conditions which contain various fractional derivatives and Riemann-Stieltjes integrals. In the proof of our main results, we use some theorems from the fixed point theory.
\end{abstract}

MSC: $34 \mathrm{~A} 08 ; 45 \mathrm{G} 15$

Keywords: Systems of Riemann-Liouville fractional differential equations; Fractional integrals; Coupled nonlocal boundary conditions; Existence of solutions

\section{Introduction}

We consider the nonlinear system of fractional differential equations

$$
\begin{cases}D_{0+}^{\alpha} x(t)+f\left(t, x(t), y(t), I_{0+}^{\theta_{1}} x(t), I_{0+}^{\sigma_{1}} y(t)\right)=0, & t \in(0,1), \\ D_{0+}^{\beta} y(t)+g\left(t, x(t), y(t), I_{0+}^{\theta_{2}} x(t), I_{0+}^{\sigma_{2}} y(t)\right)=0, & t \in(0,1),\end{cases}
$$

with the coupled nonlocal boundary conditions

$$
\begin{cases}x(0)=x^{\prime}(0)=\cdots=x^{(n-2)}(0)=0, & D_{0+}^{\gamma_{0}} x(1)=\sum_{i=1}^{p} \int_{0}^{1} D_{0+}^{\gamma_{i}} y(t) d H_{i}(t), \\ y(0)=y^{\prime}(0)=\cdots=y^{(m-2)}(0)=0, & D_{0+}^{\delta_{0}} y(1)=\sum_{i=1}^{q} \int_{0}^{1} D_{0+}^{\delta_{i}} x(t) d K_{i}(t),\end{cases}
$$

where $\alpha, \beta \in \mathbb{R}, \alpha \in(n-1, n], \beta \in(m-1, m], n, m \in \mathbb{N}, n \geq 2, m \geq 2, \theta_{1}, \theta_{2}, \sigma_{1}, \sigma_{2}>0$, $p, q \in \mathbb{N}, \gamma_{i} \in \mathbb{R}$ for all $i=0, \ldots, p, 0 \leq \gamma_{1}<\gamma_{2}<\cdots<\gamma_{p}<\beta-1, \gamma_{0} \in[0, \alpha-1), \delta_{i} \in \mathbb{R}$ for all $i=0, \ldots, q, 0 \leq \delta_{1}<\delta_{2}<\cdots<\delta_{q}<\alpha-1, \delta_{0} \in[0, \beta-1), D_{0_{+}}^{k}$ denotes the Riemann-Liouville derivative of order $k$ (for $k=\alpha, \beta, \gamma_{0}, \gamma_{i}, i=1, \ldots, p, \delta_{0}, \delta_{i}, i=1, \ldots, q$ ), $I_{0+}^{\zeta}$ is the RiemannLiouville integral of order $\zeta$ (for $\zeta=\theta_{1}, \sigma_{1}, \theta_{2}, \sigma_{2}$ ), $f$ and $g$ are nonlinear functions, and the integrals from the boundary conditions $(B C)$ are Riemann-Stieltjes integrals with $H_{i}$ for $i=1, \ldots, p$ and $K_{i}$ for $i=1, \ldots, q$ functions of bounded variation.

(c) The Author(s) 2021. This article is licensed under a Creative Commons Attribution 4.0 International License, which permits use, sharing, adaptation, distribution and reproduction in any medium or format, as long as you give appropriate credit to the original author(s) and the source, provide a link to the Creative Commons licence, and indicate if changes were made. The images or other third party material in this article are included in the article's Creative Commons licence, unless indicated otherwise in a credit line to the material. If material is not included in the article's Creative Commons licence and your intended use is not permitted by statutory regulation or exceeds the permitted use, you will need to obtain permission directly from the copyright holder. To view a copy of this licence, visit http://creativecommons.org/licenses/by/4.0/. 
In this paper, we show the existence and uniqueness of solutions for problem $(S)-(B C)$, by applying standard fixed point theorems. We prove the existence of a unique solution by using the Banach contraction mapping principle, and five existence results by applying the Leray-Schauder alternative theorem, the Krasnosel'skii theorem for the sum of two operators (for two results), the Schauder fixed point theorem, and the nonlinear alternative of Leray-Schauder-type, respectively. The methods used for proofs are standard, but their applications in this framework of systems of coupled Riemann-Liouville fractional boundary value problems are new.

In the last decades, many authors investigated the existence of positive solutions for Riemann-Liouville fractional differential equations and systems of Riemann-Liouville fractional differential equations, subject to nonlocal boundary conditions. For example, the existence and multiplicity of positive solutions for the equation

$$
D_{0+}^{\alpha} u(t)+f(t, u(t))=0, \quad t \in(0,1)
$$

with the nonlocal boundary conditions

$$
u(0)=u^{\prime}(0)=\cdots=u^{(n-2)}(0)=0, \quad D_{0+}^{\beta_{0}} u(1)=\sum_{i=1}^{m} \int_{0}^{1} D_{0+}^{\beta_{i}} u(t) d H_{i}(t)
$$

where $\alpha \in \mathbb{R}, \alpha \in(n-1, n], n, m \in \mathbb{N}, n \geq 3, \beta_{i} \in \mathbb{R}$ for all $i=0, \ldots, m, 0 \leq \beta_{1}<\beta_{2}<\cdots<$ $\beta_{m} \leq \beta_{0}<\alpha-1$, and where the function $f$ may change sign and be singular in the points $t=0,1$ and/or in the space variable $u$, was studied in the paper [1]. In the proof of the main results of [1], the authors used various height functions of the nonlinearity of the equation defined on special bounded sets, some properties of the corresponding Green functions, and two theorems from the fixed point index theory. Equation $(E)$ with a positive parameter $\lambda$, supplemented with the boundary conditions

$$
u(0)=u^{\prime}(0)=\cdots=u^{(n-2)}(0)=0, \quad D_{0+}^{p} u(1)=\sum_{i=1}^{m} a_{i} D_{0+}^{q} u\left(\xi_{i}\right),
$$

where $\xi_{i} \in \mathbb{R}, i=1, \ldots, m, 0<\xi_{1}<\cdots<\xi_{m}<1, p, q \in \mathbb{R}, p \in[1, n-2], q \in[0, p]$, was investigated in [16]. In this paper, the nonlinearity $f$ changes sign and it is singular only for $t=0,1$, while the authors used the Guo-Krasnosel'skii fixed point theorem to prove the existence of positive solutions when the parameter belongs to various intervals. For some recent results on the existence, nonexistence, and multiplicity of solutions for fractional differential equations and systems of fractional differential equations subject to various boundary conditions, we refer the reader to the monographs $[15,38]$ and the papers [1$6,14,17-19,23-25,30,35-37]$. We also mention the books [8-10, 20, 21, 29, 31, 32], and the papers $[7,11-13,26-28,33]$, for applications of the fractional differential equations in various disciplines.

The main features of the present work are the following. Firstly, the system and the coupled boundary conditions contain Riemann-Liouville fractional derivatives, and secondly, the nonlinearities in the system depend not only on the unknown functions $x$ and $y$, but also on the Riemann-Liouville fractional integrals of $x$ and $y$. Thirdly, the obtained solution $(x, y)$ is a general one which can change sign. Section 2 contains an auxiliary lemma 
which is important to establish our main theorems, some notations, and the operator associated to our problem. The main existence results are presented in Sect. 3, and in Sect. 4 we give some illustrative examples.

\section{Auxiliary results}

We consider here the system of fractional differential equations

$$
\begin{cases}D_{0+}^{\alpha} x(t)+h(t)=0, & t \in(0,1), \\ D_{0+}^{\beta} y(t)+k(t)=0, & t \in(0,1),\end{cases}
$$

with the boundary conditions $(B C)$, where $h, k \in C(0,1) \cap L^{1}(0,1)$. We denote by

$$
\begin{aligned}
& \Delta_{1}=\sum_{i=1}^{p} \frac{\Gamma(\beta)}{\Gamma\left(\beta-\gamma_{i}\right)} \int_{0}^{1} s^{\beta-\gamma_{i}-1} d H_{i}(s), \quad \Delta_{2}=\sum_{i=1}^{q} \frac{\Gamma(\alpha)}{\Gamma\left(\alpha-\delta_{i}\right)} \int_{0}^{1} s^{\alpha-\delta_{i}-1} d K_{i}(s), \\
& \Delta=\frac{\Gamma(\alpha) \Gamma(\beta)}{\Gamma\left(\alpha-\gamma_{0}\right) \Gamma\left(\beta-\delta_{0}\right)}-\Delta_{1} \Delta_{2} .
\end{aligned}
$$

By using similar arguments as those used in the proof of Lemma 2.1 from [34], we obtain the following result.

Lemma 2.1 If $\Delta \neq 0$, then the unique solution $(x, y) \in C[0,1] \times C[0,1]$ of problem $(1)-(B C)$ is given by

$$
\begin{aligned}
x(t)= & -\frac{1}{\Gamma(\alpha)} \int_{0}^{t}(t-s)^{\alpha-1} h(s) d s \\
& +\frac{t^{\alpha-1}}{\Delta}\left[\frac{\Gamma(\beta)}{\Gamma\left(\alpha-\gamma_{0}\right) \Gamma\left(\beta-\delta_{0}\right)} \int_{0}^{1}(1-s)^{\alpha-\gamma_{0}-1} h(s) d s\right. \\
& -\frac{\Gamma(\beta)}{\Gamma\left(\beta-\delta_{0}\right)} \sum_{i=1}^{p} \frac{1}{\Gamma\left(\beta-\gamma_{i}\right)} \int_{0}^{1}\left(\int_{0}^{s}(s-\tau)^{\beta-\gamma_{i}-1} k(\tau) d \tau\right) d H_{i}(s) \\
& +\frac{\Delta_{1}}{\Gamma\left(\beta-\delta_{0}\right)} \int_{0}^{1}(1-s)^{\beta-\delta_{0}-1} k(s) d s \\
& \left.-\Delta_{1}\left(\sum_{i=1}^{q} \frac{1}{\Gamma\left(\alpha-\delta_{i}\right)} \int_{0}^{1}\left(\int_{0}^{s}(s-\tau)^{\alpha-\delta_{i}-1} h(\tau) d \tau\right) d K_{i}(s)\right)\right] \\
& t \in[0,1], \\
y(t)= & -\frac{1}{\Gamma(\beta)} \int_{0}^{t}(t-s)^{\beta-1} k(s) d s \\
& +\frac{t^{\beta-1}}{\Delta}\left[\frac{\Gamma(\alpha)}{\Gamma\left(\alpha-\gamma_{0}\right) \Gamma\left(\beta-\delta_{0}\right)} \int_{0}^{1}(1-s)^{\beta-\delta_{0}-1} k(s) d s\right. \\
& -\frac{\Gamma(\alpha)}{\Gamma\left(\alpha-\gamma_{0}\right)} \sum_{i=1}^{q} \frac{1}{\Gamma\left(\alpha-\delta_{i}\right)} \int_{0}^{1}\left(\int_{0}^{s}(s-\tau)^{\alpha-\delta_{i}-1} h(\tau) d \tau\right) d K_{i}(s) \\
& +\frac{\Delta_{2}}{\Gamma\left(\alpha-\gamma_{0}\right)} \int_{0}^{1}(1-s)^{\alpha-\gamma_{0}-1} h(s) d s
\end{aligned}
$$




$$
\left.-\Delta_{2}\left(\sum_{i=1}^{p} \frac{1}{\Gamma\left(\beta-\gamma_{i}\right)} \int_{0}^{1}\left(\int_{0}^{s}(s-\tau)^{\beta-\gamma_{i}-1} k(\tau) d \tau\right) d H_{i}(s)\right)\right], \quad t \in[0,1]
$$

Remark 2.1 If $u \in C[0,1]$ then for $\chi>0$ we have

$$
\left|I_{0+}^{\chi} u(t)\right| \leq \frac{\|u\|}{\Gamma(\chi+1)}, \quad \forall t \in[0,1]
$$

where $\|u\|=\sup _{t \in[0,1]}|u(t)|$.

We introduce now the assumption $(J 1)$ for problem $(S)-(B C)$ that will be used in our main results.

(J1) $\alpha, \beta \in \mathbb{R}, \alpha \in(n-1, n], \beta \in(m-1, m], n, m \in \mathbb{N}, n \geq 2, m \geq 2, \theta_{1}, \theta_{2}, \sigma_{1}, \sigma_{2}>0, p, q \in$ $\mathbb{N}, \gamma_{i} \in \mathbb{R}$ for all $i=0, \ldots, p, 0 \leq \gamma_{1}<\gamma_{2}<\cdots<\gamma_{p}<\beta-1, \gamma_{0} \in[0, \beta-1), \delta_{i} \in \mathbb{R}$ for all $i=0, \ldots, q, 0 \leq \delta_{1}<\delta_{2}<\cdots<\delta_{q}<\alpha-1, \delta_{0} \in[0, \alpha-1), H_{i}:[0,1] \rightarrow \mathbb{R}, i=1, \ldots, p$ and $K_{j}:[0,1] \rightarrow \mathbb{R}, j=1, \ldots, q$ are functions of bounded variation, and $\Delta \neq 0$.

We introduce the following constants:

$$
\begin{aligned}
& M_{1}=1+\frac{1}{\Gamma\left(\theta_{1}+1\right)}, \quad M_{2}=1+\frac{1}{\Gamma\left(\sigma_{1}+1\right)}, \quad M_{3}=1+\frac{1}{\Gamma\left(\theta_{2}+1\right)} \\
& M_{4}=1+\frac{1}{\Gamma\left(\sigma_{2}+1\right)}, \quad M_{5}=\max \left\{M_{1}, M_{2}\right\}, \quad M_{6}=\max \left\{M_{3}, M_{4}\right\} \\
& M_{7}=\frac{1}{\Gamma(\alpha+1)}+\frac{\Gamma(\beta)}{|\Delta| \Gamma\left(\alpha-\gamma_{0}+1\right) \Gamma\left(\beta-\delta_{0}\right)} \\
& +\frac{1}{|\Delta|}\left(\sum_{i=1}^{p} \frac{\Gamma(\beta)}{\Gamma\left(\beta-\gamma_{i}\right)}\left|\int_{0}^{1} s^{\beta-\gamma_{i}-1} d H_{i}(s)\right|\right) \\
& \times\left(\sum_{i=1}^{q} \frac{1}{\Gamma\left(\alpha-\delta_{i}+1\right)}\left|\int_{0}^{1} s^{\alpha-\delta_{i}} d K_{i}(s)\right|\right) \\
& M_{8}=\frac{1}{\Gamma(\beta+1)}+\frac{\Gamma(\alpha)}{|\Delta| \Gamma\left(\alpha-\gamma_{0}\right) \Gamma\left(\beta-\delta_{0}+1\right)} \\
& +\frac{1}{|\Delta|}\left(\sum_{i=1}^{q} \frac{\Gamma(\alpha)}{\Gamma\left(\alpha-\delta_{i}\right)}\left|\int_{0}^{1} s^{\alpha-\delta_{i}-1} d K_{i}(s)\right|\right) \\
& \times\left(\sum_{i=1}^{p} \frac{1}{\Gamma\left(\beta-\gamma_{i}+1\right)}\left|\int_{0}^{1} s^{\beta-\gamma_{i}} d H_{i}(s)\right|\right) \\
& M_{9}=\frac{\Gamma(\alpha)}{|\Delta| \Gamma\left(\alpha-\gamma_{0}\right)} \sum_{i=1}^{q} \frac{1}{\Gamma\left(\alpha-\delta_{i}+1\right)}\left|\int_{0}^{1} s^{\alpha-\delta_{i}} d K_{i}(s)\right| \\
& +\frac{1}{|\Delta| \Gamma\left(\alpha-\gamma_{0}+1\right)}\left(\sum_{i=1}^{q} \frac{\Gamma(\alpha)}{\Gamma\left(\alpha-\delta_{i}\right)}\left|\int_{0}^{1} s^{\alpha-\delta_{i}-1} d K_{i}(s)\right|\right) \\
& M_{10}=\frac{\Gamma(\beta)}{|\Delta| \Gamma\left(\beta-\delta_{0}\right)} \sum_{i=1}^{p} \frac{1}{\Gamma\left(\beta-\gamma_{i}+1\right)}\left|\int_{0}^{1} s^{\beta-\gamma_{i}} d H_{i}(s)\right| \\
& +\frac{1}{|\Delta| \Gamma\left(\beta-\delta_{0}+1\right)}\left(\sum_{i=1}^{p} \frac{\Gamma(\beta)}{\Gamma\left(\beta-\gamma_{i}\right)}\left|\int_{0}^{1} s^{\beta-\gamma_{i}-1} d H_{i}(s)\right|\right),
\end{aligned}
$$




$$
M_{11}=M_{7}-\frac{1}{\Gamma(\alpha+1)}, \quad M_{12}=M_{8}-\frac{1}{\Gamma(\beta+1)} .
$$

We consider the Banach space $X=C[0,1]$ with the supremum norm $\|x\|=\sup _{t \in[0,1]}|x(t)|$, and the Banach space $Y=X \times X$ with the norm $\|(x, y)\|_{Y}=\|x\|+\|y\|$. We introduce the operator $Q: Y \rightarrow Y$ defined by $Q(u, v)=\left(Q_{1}(u, v), Q_{2}(u, v)\right)$ for $(u, v) \in Y$, where the operators $Q_{1}, Q_{2}: Y \rightarrow X$ are given by

$$
\begin{aligned}
& Q_{1}(x, y)(t)=-\frac{1}{\Gamma(\alpha)} \int_{0}^{t}(t-s)^{\alpha-1} \hat{f}_{x y}(s) d s \\
& +\frac{t^{\alpha-1} \Gamma(\beta)}{\Delta \Gamma\left(\alpha-\gamma_{0}\right) \Gamma\left(\beta-\delta_{0}\right)} \int_{0}^{1}(1-s)^{\alpha-\gamma_{0}-1} \hat{f}_{x y}(s) d s \\
& -\frac{t^{\alpha-1} \Gamma(\beta)}{\Delta \Gamma\left(\beta-\delta_{0}\right)} \sum_{i=1}^{p} \frac{1}{\Gamma\left(\beta-\gamma_{i}\right)} \int_{0}^{1}\left(\int_{0}^{s}(s-\tau)^{\beta-\gamma_{i}-1} \hat{g}_{x y}(\tau) d \tau\right) d H_{i}(s) \\
& +\frac{t^{\alpha-1} \Delta_{1}}{\Delta \Gamma\left(\beta-\delta_{0}\right)} \int_{0}^{1}(1-s)^{\beta-\delta_{0}-1} \hat{g}_{x y}(s) d s \\
& -\frac{t^{\alpha-1} \Delta_{1}}{\Delta}\left(\sum_{i=1}^{q} \frac{1}{\Gamma\left(\alpha-\delta_{i}\right)} \int_{0}^{1}\left(\int_{0}^{s}(s-\tau)^{\alpha-\delta_{i}-1} \hat{f}_{x y}(\tau) d \tau\right) d K_{i}(s)\right), \\
& Q_{2}(x, y)(t)=-\frac{1}{\Gamma(\beta)} \int_{0}^{t}(t-s)^{\beta-1} \hat{g}_{x y}(s) d s \\
& +\frac{t^{\beta-1} \Gamma(\alpha)}{\Delta \Gamma\left(\alpha-\gamma_{0}\right) \Gamma\left(\beta-\delta_{0}\right)} \int_{0}^{1}(1-s)^{\beta-\delta_{0}-1} \hat{g}_{x y}(s) d s \\
& -\frac{t^{\beta-1} \Gamma(\alpha)}{\Delta \Gamma\left(\alpha-\gamma_{0}\right)} \sum_{i=1}^{q} \frac{1}{\Gamma\left(\alpha-\delta_{i}\right)} \int_{0}^{1}\left(\int_{0}^{s}(s-\tau)^{\alpha-\delta_{i}-1} \hat{f}_{x y}(\tau) d \tau\right) d K_{i}(s) \\
& +\frac{t^{\beta-1} \Delta_{2}}{\Delta \Gamma\left(\alpha-\gamma_{0}\right)} \int_{0}^{1}(1-s)^{\alpha-\gamma_{0}-1} \hat{f}_{x y}(s) d s \\
& -\frac{t^{\beta-1} \Delta_{2}}{\Delta}\left(\sum_{i=1}^{p} \frac{1}{\Gamma\left(\beta-\gamma_{i}\right)} \int_{0}^{1}\left(\int_{0}^{s}(s-\tau)^{\beta-\gamma_{i}-1} \hat{g}_{x y}(\tau) d \tau\right) d H_{i}(s)\right),
\end{aligned}
$$

for $t \in[0,1]$ and $(x, y) \in Y$, where $\hat{f}_{x y}(s)=f\left(s, x(s), y(s), I_{0+}^{\theta_{1}} x(s), I_{0+}^{\sigma_{1}} y(s)\right), \hat{g}_{x y}(s)=g(s, x(s), y(s)$, $\left.I_{0+}^{\theta_{2}} x(s), I_{0+}^{\sigma_{2}} y(s)\right)$ for $s \in[0,1]$.

By using Lemma 2.1 , we see that $(x, y)$ is a solution of problem $(S)-(B C)$ if and only if $(x, y)$ is a fixed point of operator $Q$.

\section{Existence of solutions}

In this section we will give some existence results for the solutions of our problem $(S)$ $(B C)$.

Theorem 3.1 Assume that (J1) and

(J2) The functions $f, g:[0,1] \times \mathbb{R}^{4} \rightarrow \mathbb{R}$ are continuous and there exist $L_{1}, L_{2}>0$ such that

$$
\left|f\left(t, u_{1}, u_{2}, u_{3}, u_{4}\right)-f\left(t, v_{1}, v_{2}, v_{3}, v_{4}\right)\right| \leq L_{1} \sum_{i=1}^{4}\left|u_{i}-v_{i}\right|,
$$




$$
\left|g\left(t, u_{1}, u_{2}, u_{3}, u_{4}\right)-g\left(t, v_{1}, v_{2}, v_{3}, v_{4}\right)\right| \leq L_{2} \sum_{i=1}^{4}\left|u_{i}-v_{i}\right|
$$

for all $t \in[0,1], u_{i}, v_{i} \in \mathbb{R}, i=1, \ldots, 4$,

hold. If $\Xi:=L_{1} M_{5}\left(M_{7}+M_{9}\right)+L_{2} M_{6}\left(M_{8}+M_{10}\right)<1$, then problem $(S)-(B C)$ has a unique solution $(x(t), y(t)), t \in[0,1]$, where $M_{5}, \ldots, M_{10}$ are given by (3).

Proof We consider the positive number $r$ given by

$$
r=\left[M_{0}\left(M_{7}+M_{9}\right)+\widetilde{M}_{0}\left(M_{8}+M_{10}\right)\right]\left[1-L_{1} M_{5}\left(M_{7}+M_{9}\right)-L_{2} M_{6}\left(M_{8}+M_{10}\right)\right]^{-1},
$$

where $M_{0}=\sup _{t \in[0,1]}|f(t, 0,0,0,0)|, \widetilde{M}_{0}=\sup _{t \in[0,1]}|g(t, 0,0,0,0)|$. We define the set $\bar{B}_{r}=$ $\left\{(x, y) \in Y,\|(x, y)\|_{Y} \leq r\right\}$ and show firstly that $Q\left(\bar{B}_{r}\right) \subset \bar{B}_{r}$. Let $(x, y) \in \bar{B}_{r}$. By using $(J 2)$ and Remark 2.1, for $\hat{f}_{x y}(t)$ we deduce the following inequalities:

$$
\begin{aligned}
\left|\hat{f}_{x y}(t)\right| & \leq\left|f\left(t, x(t), y(t), I_{0+}^{\theta_{1}} x(t), I_{0+}^{\sigma_{1}} y(t)\right)-f(t, 0,0,0,0)\right|+|f(t, 0,0,0,0)| \\
& \leq L_{1}\left(|x(t)|+|y(t)|+\left|I_{0+}^{\theta_{1}} x(t)\right|+\left|I_{0+}^{\sigma_{1}} y(t)\right|\right)+M_{0} \\
& \leq L_{1}\left(\|x\|+\|y\|+\frac{\|x\|}{\Gamma\left(\theta_{1}+1\right)}+\frac{\|y\|}{\Gamma\left(\sigma_{1}+1\right)}\right)+M_{0} \\
& =L_{1}\left(M_{1}\|x\|+M_{2}\|y\|\right)+M_{0} \\
& \leq L_{1} M_{5}\|(x, y)\|_{Y}+M_{0} \leq L_{1} M_{5} r+M_{0}, \quad \forall t \in[0,1] .
\end{aligned}
$$

Arguing as before, we find

$$
\begin{aligned}
\left|\hat{g}_{x y}(t)\right| & \leq\left|g\left(t, x(t), y(t), I_{0+}^{\theta_{2}} x(t), I_{0+}^{\sigma_{2}} y(t)\right)-g(t, 0,0,0,0)\right|+|g(t, 0,0,0,0)| \\
& \leq L_{2}\left(|x(t)|+|y(t)|+\left|I_{0+}^{\theta_{2}} x(t)\right|+\left|I_{0+}^{\sigma_{2}} y(t)\right|\right)+\widetilde{M}_{0} \\
& \leq L_{2}\left(\|x\|+\|y\|+\frac{\|x\|}{\Gamma\left(\theta_{2}+1\right)}+\frac{\|y\|}{\Gamma\left(\sigma_{2}+1\right)}\right)+\widetilde{M}_{0} \\
& =L_{2}\left(M_{3}\|x\|+M_{4}\|y\|\right)+\widetilde{M}_{0} \\
& \leq L_{2} M_{6}\|(x, y)\|_{Y}+\widetilde{M}_{0} \leq L_{2} M_{6} r+\widetilde{M}_{0}, \quad \forall t \in[0,1] .
\end{aligned}
$$

Then by the definition of operators $Q_{1}$ and $Q_{2}$, we conclude

$$
\begin{aligned}
& \left|Q_{1}(x, y)(t)\right| \\
& \leq \frac{1}{\Gamma(\alpha)} \int_{0}^{t}(t-s)^{\alpha-1}\left(L_{1} M_{5} r+M_{0}\right) d s \\
& \quad+\frac{t^{\alpha-1} \Gamma(\beta)}{|\Delta| \Gamma\left(\alpha-\gamma_{0}\right) \Gamma\left(\beta-\delta_{0}\right)} \int_{0}^{1}(1-s)^{\alpha-\gamma_{0}-1}\left(L_{1} M_{5} r+M_{0}\right) d s \\
& \quad+\frac{t^{\alpha-1} \Gamma(\beta)}{|\Delta| \Gamma\left(\beta-\delta_{0}\right)} \sum_{i=1}^{p} \frac{1}{\Gamma\left(\beta-\gamma_{i}\right)}\left|\int_{0}^{1}\left(\int_{0}^{s}(s-\tau)^{\beta-\gamma_{i}-1}\left(L_{2} M_{6} r+\widetilde{M}_{0}\right) d \tau\right) d H_{i}(s)\right| \\
& \quad+\frac{t^{\alpha-1}}{|\Delta|}\left(\sum_{i=1}^{p} \frac{\Gamma(\beta)}{\Gamma\left(\beta-\gamma_{i}\right)}\left|\int_{0}^{1} s^{\beta-\gamma_{i}-1} d H_{i}(s)\right|\right)
\end{aligned}
$$


Luck Advances in Difference Equations

(2021) 2021:134

Page 7 of 25

$$
\begin{aligned}
& \times\left(\frac{1}{\Gamma\left(\beta-\delta_{0}\right)} \int_{0}^{1}(1-s)^{\beta-\delta_{0}-1}\left(L_{2} M_{6} r+\tilde{M}_{0}\right) d s\right) \\
& +\frac{t^{\alpha-1}}{|\Delta|}\left(\sum_{i=1}^{p} \frac{\Gamma(\beta)}{\Gamma\left(\beta-\gamma_{i}\right)}\left|\int_{0}^{1} s^{\beta-\gamma_{i}-1} d H_{i}(s)\right|\right) \\
& \times\left(\sum_{i=1}^{q} \frac{1}{\Gamma\left(\alpha-\delta_{i}\right)}\left|\int_{0}^{1}\left(\int_{0}^{s}(s-\tau)^{\alpha-\delta_{i}-1}\left(L_{1} M_{5} r+M_{0}\right) d \tau\right) d K_{i}(s)\right|\right) \\
& =\left(L_{1} M_{5} r+M_{0}\right)\left[\frac{1}{\Gamma(\alpha)} \int_{0}^{t}(t-s)^{\alpha-1} d s+\frac{t^{\alpha-1} \Gamma(\beta)}{|\Delta| \Gamma\left(\alpha-\gamma_{0}\right) \Gamma\left(\beta-\delta_{0}\right)} \int_{0}^{1}(1-s)^{\alpha-\gamma_{0}-1} d s\right. \\
& +\frac{t^{\alpha-1}}{|\Delta|}\left(\sum_{i=1}^{p} \frac{\Gamma(\beta)}{\Gamma\left(\beta-\gamma_{i}\right)}\left|\int_{0}^{1} s^{\beta-\gamma_{i}-1} d H_{i}(s)\right|\right) \\
& \left.\times\left(\sum_{i=1}^{q} \frac{1}{\Gamma\left(\alpha-\delta_{i}\right)}\left|\int_{0}^{1}\left(\int_{0}^{s}(s-\tau)^{\alpha-\delta_{i}-1} d \tau\right) d K_{i}(s)\right|\right)\right] \\
& +\left(L_{2} M_{6} r+\tilde{M}_{0}\right)\left[\frac{t^{\alpha-1} \Gamma(\beta)}{|\Delta| \Gamma\left(\beta-\delta_{0}\right)} \sum_{i=1}^{p} \frac{1}{\Gamma\left(\beta-\gamma_{i}\right)}\left|\int_{0}^{1}\left(\int_{0}^{s}(s-\tau)^{\beta-\gamma_{i}-1} d \tau\right) d H_{i}(s)\right|\right. \\
& \left.+\frac{t^{\alpha-1}}{|\Delta|}\left(\sum_{i=1}^{p} \frac{\Gamma(\beta)}{\Gamma\left(\beta-\gamma_{i}\right)}\left|\int_{0}^{1} s^{\beta-\gamma_{i}-1} d H_{i}(s)\right|\right)\left(\frac{1}{\Gamma\left(\beta-\delta_{0}\right)} \int_{0}^{1}(1-s)^{\beta-\delta_{0}-1} d s\right)\right] \\
& =\left(L_{1} M_{5} r+M_{0}\right)\left[\frac{t^{\alpha}}{\Gamma(\alpha+1)}+\frac{t^{\alpha-1} \Gamma(\beta)}{|\Delta| \Gamma\left(\alpha-\gamma_{0}+1\right) \Gamma\left(\beta-\delta_{0}\right)}\right. \\
& \left.+\frac{t^{\alpha-1}}{|\Delta|}\left(\sum_{i=1}^{p} \frac{\Gamma(\beta)}{\Gamma\left(\beta-\gamma_{i}\right)}\left|\int_{0}^{1} s^{\beta-\gamma_{i}-1} d H_{i}(s)\right|\right)\left(\sum_{i=1}^{q} \frac{1}{\Gamma\left(\alpha-\delta_{i}+1\right)}\left|\int_{0}^{1} s^{\alpha-\delta_{i}} d K_{i}(s)\right|\right)\right] \\
& +\left(L_{2} M_{6} r+\tilde{M}_{0}\right)\left[\frac{t^{\alpha-1} \Gamma(\beta)}{|\Delta| \Gamma\left(\beta-\delta_{0}\right)} \sum_{i=1}^{p} \frac{1}{\Gamma\left(\beta-\gamma_{i}+1\right)}\left|\int_{0}^{1} s^{\beta-\gamma_{i}} d H_{i}(s)\right|\right. \\
& \left.+\frac{t^{\alpha-1}}{|\Delta| \Gamma\left(\beta-\delta_{0}+1\right)}\left(\sum_{i=1}^{p} \frac{\Gamma(\beta)}{\Gamma\left(\beta-\gamma_{i}\right)}\left|\int_{0}^{1} s^{\beta-\gamma_{i}-1} d H_{i}(s)\right|\right)\right], \quad \forall t \in[0,1] .
\end{aligned}
$$

Therefore we obtain

$$
\begin{aligned}
& \left\|Q_{1}(x, y)\right\| \\
& \leq \quad\left(L_{1} M_{5} r+M_{0}\right)\left[\frac{1}{\Gamma(\alpha+1)}+\frac{\Gamma(\beta)}{|\Delta| \Gamma\left(\alpha-\gamma_{0}+1\right) \Gamma\left(\beta-\delta_{0}\right)}\right. \\
& \quad+\frac{1}{|\Delta|}\left(\sum_{i=1}^{p} \frac{\Gamma(\beta)}{\Gamma\left(\beta-\gamma_{i}\right)}\left|\int_{0}^{1} s^{\beta-\gamma_{i}-1} d H_{i}(s)\right|\right) \\
& \left.\quad \times\left(\sum_{i=1}^{q} \frac{1}{\Gamma\left(\alpha-\delta_{i}+1\right)}\left|\int_{0}^{1} s^{\alpha-\delta_{i}} d K_{i}(s)\right|\right)\right] \\
& \quad+\left(L_{2} M_{6} r+\tilde{M}_{0}\right)\left[\frac{\Gamma(\beta)}{|\Delta| \Gamma\left(\beta-\delta_{0}\right)} \sum_{i=1}^{p} \frac{1}{\Gamma\left(\beta-\gamma_{i}+1\right)}\left|\int_{0}^{1} s^{\beta-\gamma_{i}} d H_{i}(s)\right|\right.
\end{aligned}
$$




$$
\begin{aligned}
& \left.+\frac{1}{|\Delta| \Gamma\left(\beta-\delta_{0}+1\right)}\left(\sum_{i=1}^{p} \frac{\Gamma(\beta)}{\Gamma\left(\beta-\gamma_{i}\right)}\left|\int_{0}^{1} s^{\beta-\gamma_{i}-1} d H_{i}(s)\right|\right)\right] \\
= & \left(L_{1} M_{5} r+M_{0}\right) M_{7}+\left(L_{2} M_{6} r+\tilde{M}_{0}\right) M_{10} .
\end{aligned}
$$

In a similar manner, we deduce

$$
\begin{aligned}
& \left\|Q_{2}(x, y)\right\| \\
& \leq\left(L_{1} M_{5} r+M_{0}\right)\left[\frac{\Gamma(\alpha)}{|\Delta| \Gamma\left(\alpha-\gamma_{0}\right)} \sum_{i=1}^{q} \frac{1}{\Gamma\left(\alpha-\delta_{i}+1\right)}\left|\int_{0}^{1} s^{\alpha-\delta_{i}} d K_{i}(s)\right|\right. \\
& \left.\quad+\frac{1}{|\Delta| \Gamma\left(\alpha-\gamma_{0}+1\right)}\left(\sum_{i=1}^{q} \frac{\Gamma(\alpha)}{\Gamma\left(\alpha-\delta_{i}\right)}\left|\int_{0}^{1} s^{\alpha-\delta_{i}-1} d K_{i}(s)\right|\right)\right] \\
& \quad+\left(L_{2} M_{6} r+\widetilde{M}_{0}\right)\left[\frac{1}{\Gamma(\beta+1)}+\frac{\Gamma(\alpha)}{|\Delta| \Gamma\left(\alpha-\gamma_{0}\right) \Gamma\left(\beta-\delta_{0}+1\right)}\right. \\
& \quad+\frac{1}{|\Delta|}\left(\sum_{i=1}^{q} \frac{\Gamma(\alpha)}{\Gamma\left(\alpha-\delta_{i}\right)}\left|\int_{0}^{1} s^{\alpha-\delta_{i}-1} d K_{i}(s)\right|\right) \\
& \left.\quad \times\left(\sum_{i=1}^{p} \frac{1}{\Gamma\left(\beta-\gamma_{i}+1\right)}\left|\int_{0}^{1} s^{\beta-\gamma_{i}} d H_{i}(s)\right|\right)\right] \\
& =\left(L_{1} M_{5} r+M_{0}\right) M_{9}+\left(L_{2} M_{6} r+\widetilde{M}_{0}\right) M_{8} .
\end{aligned}
$$

By relations (5) and (6), we conclude

$$
\begin{aligned}
\|Q(x, y)\|_{Y} & =\left\|Q_{1}(x, y)\right\|+\left\|Q_{2}(x, y)\right\| \\
& \leq\left(L_{1} M_{5} r+M_{0}\right)\left(M_{7}+M_{9}\right)+\left(L_{2} M_{6} r+\widetilde{M}_{0}\right)\left(M_{8}+M_{10}\right)=r,
\end{aligned}
$$

for all $(x, y) \in \bar{B}_{r}$, which implies that $Q\left(\bar{B}_{r}\right) \subset \bar{B}_{r}$.

Next we prove that operator $Q$ is a contraction. For $\left(x_{i}, y_{i}\right) \in \bar{B}_{r}, i=1,2$, and for each $t \in[0,1]$, we have

$$
\begin{aligned}
& \left|Q_{1}\left(x_{1}, y_{1}\right)(t)-Q_{1}\left(x_{2}, y_{2}\right)(t)\right| \\
& \leq \frac{1}{\Gamma(\alpha)} \int_{0}^{t}(t-s)^{\alpha-1}\left|\hat{f}_{x_{1} y_{1}}(s)-\hat{f}_{x_{2} y_{2}}(s)\right| d s \\
& \quad+\frac{t^{\alpha-1} \Gamma(\beta)}{|\Delta| \Gamma\left(\alpha-\gamma_{0}\right) \Gamma\left(\beta-\delta_{0}\right)} \int_{0}^{1}(1-s)^{\alpha-\gamma_{0}-1}\left|\hat{f}_{x_{1} y_{1}}(s)-\hat{f}_{x_{2} y_{2}}(s)\right| d s \\
& \quad+\frac{t^{\alpha-1} \Gamma(\beta)}{|\Delta| \Gamma\left(\beta-\delta_{0}\right)} \sum_{i=1}^{p} \frac{1}{\Gamma\left(\beta-\gamma_{i}\right)} \\
& \quad \times\left|\int_{0}^{1}\left(\int_{0}^{s}(s-\tau)^{\beta-\gamma_{i}-1}\left|\hat{g}_{x_{1} y_{1}}(\tau)-\hat{g}_{x_{2} y_{2}}(\tau)\right| d \tau\right) d H_{i}(s)\right| \\
& \quad+\frac{t^{\alpha-1}}{|\Delta|}\left(\sum_{i=1}^{p} \frac{\Gamma(\beta)}{\Gamma\left(\beta-\gamma_{i}\right)}\left|\int_{0}^{1} s^{\beta-\gamma_{i}-1} d H_{i}(s)\right|\right) \\
& \quad \times\left(\frac{1}{\Gamma\left(\beta-\delta_{0}\right)} \int_{0}^{1}(1-s)^{\beta-\delta_{0}-1}\left|\hat{g}_{x_{1} y_{1}}(s)-\hat{g}_{x_{2} y_{2}}(s)\right| d s\right)
\end{aligned}
$$




$$
\begin{aligned}
& +\frac{t^{\alpha-1}}{|\Delta|}\left(\sum_{i=1}^{p} \frac{\Gamma(\beta)}{\Gamma\left(\beta-\gamma_{i}\right)}\left|\int_{0}^{1} s^{\beta-\gamma_{i}-1} d H_{i}(s)\right|\right) \\
& \times\left(\sum_{i=1}^{q} \frac{1}{\Gamma\left(\alpha-\delta_{i}\right)}\left|\int_{0}^{1}\left(\int_{0}^{s}(s-\tau)^{\alpha-\delta_{i}-1}\left|\hat{f}_{x_{1} y_{1}}(\tau)-\hat{f}_{x_{2} y_{2}}(\tau)\right| d \tau\right) d K_{i}(s)\right|\right) .
\end{aligned}
$$

\section{Because}

$$
\begin{aligned}
\left|\hat{f}_{x_{1} y_{1}}(s)-\hat{f}_{x_{2} y_{2}}(s)\right| & \\
\leq & L_{1}\left(\left|x_{1}(s)-x_{2}(s)\right|+\left|y_{1}(s)-y_{2}(s)\right|\right. \\
& \left.+\left|I_{0+}^{\theta_{1}} x_{1}(s)-I_{0+}^{\theta_{1}} x_{2}(s)\right|+\left|I_{0+}^{\sigma_{1}} y_{1}(s)-I_{0+}^{\sigma_{1}} y_{2}(s)\right|\right) \\
\leq & L_{1}\left(\left\|x_{1}-x_{2}\right\|+\left\|y_{1}-y_{2}\right\|+\frac{1}{\Gamma\left(\theta_{1}+1\right)}\left\|x_{1}-x_{2}\right\|+\frac{1}{\Gamma\left(\sigma_{1}+1\right)}\left\|y_{1}-y_{2}\right\|\right) \\
= & L_{1}\left(M_{1}\left\|x_{1}-x_{2}\right\|+M_{2}\left\|y_{1}-y_{2}\right\|\right) \leq L_{1} M_{5}\left\|\left(x_{1}, y_{1}\right)-\left(x_{2}, y_{2}\right)\right\|_{Y}, \quad \forall s \in[0,1], \\
\left|\hat{g}_{x_{1} y_{1}}(s)-\hat{g}_{x_{2} y_{2}}(s)\right| & \\
\leq & L_{2}\left(\left|x_{1}(s)-x_{2}(s)\right|+\left|y_{1}(s)-y_{2}(s)\right|\right. \\
& \left.+\left|I_{0+}^{\theta_{2}} x_{1}(s)-I_{0+}^{\theta_{2}} x_{2}(s)\right|+\left|I_{0+}^{\sigma_{2}} y_{1}(s)-I_{0+}^{\sigma_{2}} y_{2}(s)\right|\right) \\
\leq & L_{2}\left(\left\|x_{1}-x_{2}\right\|+\left\|y_{1}-y_{2}\right\|+\frac{1}{\Gamma\left(\theta_{2}+1\right)}\left\|x_{1}-x_{2}\right\|+\frac{1}{\Gamma\left(\sigma_{2}+1\right)}\left\|y_{1}-y_{2}\right\|\right) \\
= & L_{2}\left(M_{3}\left\|x_{1}-x_{2}\right\|+M_{4}\left\|y_{1}-y_{2}\right\|\right) \leq L_{2} M_{6}\left\|\left(x_{1}, y_{1}\right)-\left(x_{2}, y_{2}\right)\right\|_{Y}, \quad \forall s \in[0,1],
\end{aligned}
$$

the inequality (7) gives us

$$
\begin{aligned}
& \left|Q_{1}\left(x_{1}, y_{1}\right)(t)-Q_{1}\left(x_{2}, y_{2}\right)(t)\right| \\
& \leq \quad L_{1} M_{5}\left\|\left(x_{1}, y_{1}\right)-\left(x_{2}, y_{2}\right)\right\|_{Y} \\
& \quad \times\left[\frac{t^{\alpha}}{\Gamma(\alpha+1)}+\frac{t^{\alpha-1} \Gamma(\beta)}{|\Delta| \Gamma\left(\alpha-\gamma_{0}+1\right) \Gamma\left(\beta-\delta_{0}\right)}\right. \\
& \left.\quad+\frac{t^{\alpha-1}}{|\Delta|}\left(\sum_{i=1}^{p} \frac{\Gamma(\beta)}{\Gamma\left(\beta-\gamma_{i}\right)}\left|\int_{0}^{1} s^{\beta-\gamma_{i}-1} d H_{i}(s)\right|\right)\left(\sum_{i=1}^{q} \frac{1}{\Gamma\left(\alpha-\delta_{i}+1\right)}\left|\int_{0}^{1} s^{\alpha-\delta_{i}} d K_{i}(s)\right|\right)\right] \\
& \quad+L_{2} M_{6}\left\|\left(x_{1}, y_{1}\right)-\left(x_{2}, y_{2}\right)\right\|_{Y}\left[\frac{t^{\alpha-1} \Gamma(\beta)}{|\Delta| \Gamma\left(\beta-\delta_{0}\right)} \sum_{i=1}^{p} \frac{1}{\Gamma\left(\beta-\gamma_{i}+1\right)}\left|\int_{0}^{1} s^{\beta-\gamma_{i}} d H_{i}(s)\right|\right. \\
& \left.\quad+\frac{t^{\alpha-1}}{|\Delta| \Gamma\left(\beta-\delta_{0}+1\right)} \sum_{i=1}^{p} \frac{\Gamma(\beta)}{\Gamma\left(\beta-\gamma_{i}\right)}\left|\int_{0}^{1} s^{\beta-\gamma_{i}-1} d H_{i}(s)\right|\right], \quad \forall t \in[0,1] .
\end{aligned}
$$

Therefore we obtain

$$
\begin{aligned}
& \left\|Q_{1}\left(x_{1}, y_{1}\right)-Q_{1}\left(x_{2}, y_{2}\right)\right\| \\
& \quad \leq\left\{L _ { 1 } M _ { 5 } \left[\frac{1}{\Gamma(\alpha+1)}+\frac{\Gamma(\beta)}{|\Delta| \Gamma\left(\alpha-\gamma_{0}+1\right) \Gamma\left(\beta-\delta_{0}\right)}\right.\right.
\end{aligned}
$$




$$
\begin{aligned}
& +\frac{1}{|\Delta|}\left(\sum_{i=1}^{p} \frac{\Gamma(\beta)}{\Gamma\left(\beta-\gamma_{i}\right)}\left|\int_{0}^{1} s^{\beta-\gamma_{i}-1} d H_{i}(s)\right|\right) \\
& \left.\times\left(\sum_{i=1}^{q} \frac{1}{\Gamma\left(\alpha-\delta_{i}+1\right)}\left|\int_{0}^{1} s^{\alpha-\delta_{i}} d K_{i}(s)\right|\right)\right] \\
& +L_{2} M_{6}\left[\frac{\Gamma(\beta)}{|\Delta| \Gamma\left(\beta-\delta_{0}\right)} \sum_{i=1}^{p} \frac{1}{\Gamma\left(\beta-\gamma_{i}+1\right)}\left|\int_{0}^{1} s^{\beta-\gamma_{i}} d H_{i}(s)\right|\right. \\
& \left.\left.+\frac{1}{|\Delta| \Gamma\left(\beta-\delta_{0}+1\right)} \sum_{i=1}^{p} \frac{\Gamma(\beta)}{\Gamma\left(\beta-\gamma_{i}\right)}\left|\int_{0}^{1} s^{\beta-\gamma_{i}-1} d H_{i}(s)\right|\right]\right\}\left\|\left(x_{1}, y_{1}\right)-\left(x_{2}, y_{2}\right)\right\|_{Y} \\
& =\left(L_{1} M_{5} M_{7}+L_{2} M_{6} M_{10}\right)\left\|\left(x_{1}, y_{1}\right)-\left(x_{2}, y_{2}\right)\right\|_{Y} .
\end{aligned}
$$

In a similar manner, we deduce

$$
\begin{aligned}
\left\|Q_{2}\left(x_{1}, y_{1}\right)-Q_{2}\left(x_{2}, y_{2}\right)\right\| & \left\{L _ { 2 } M _ { 6 } \left[\frac{1}{\Gamma(\beta+1)}+\frac{\Gamma(\alpha)}{|\Delta| \Gamma\left(\alpha-\gamma_{0}\right) \Gamma\left(\beta-\delta_{0}+1\right)}\right.\right. \\
& +\frac{1}{|\Delta|}\left(\sum_{i=1}^{q} \frac{\Gamma(\alpha)}{\Gamma\left(\alpha-\delta_{i}\right)}\left|\int_{0}^{1} s^{\alpha-\delta_{i}-1} d K_{i}(s)\right|\right) \\
& \left.\times\left(\sum_{i=1}^{p} \frac{1}{\Gamma\left(\beta-\gamma_{i}+1\right)}\left|\int_{0}^{1} s^{\beta-\gamma_{i}} d H_{i}(s)\right|\right)\right] \\
& +L_{1} M_{5}\left[\frac{\Gamma(\alpha)}{|\Delta| \Gamma\left(\alpha-\gamma_{0}\right)} \sum_{i=1}^{q} \frac{1}{\Gamma\left(\alpha-\delta_{i}+1\right)}\left|\int_{0}^{1} s^{\alpha-\delta_{i}} d K_{i}(s)\right|\right. \\
& \left.\left.+\frac{1}{|\Delta| \Gamma\left(\alpha-\gamma_{0}+1\right)} \sum_{i=1}^{q} \frac{\Gamma(\alpha)}{\Gamma\left(\alpha-\delta_{i}\right)}\left|\int_{0}^{1} s^{\alpha-\delta_{i}-1} d K_{i}(s)\right|\right]\right\}\left(x_{1}, y_{1}\right)-\left(x_{2}, y_{2}\right) \|_{Y} \\
= & \left(L_{1} M_{5} M_{9}+L_{2} M_{6} M_{8}\right)\left\|\left(x_{1}, y_{1}\right)-\left(x_{2}, y_{2}\right)\right\| Y_{Y}
\end{aligned}
$$

Then by using relations (8) and (9), we obtain

$$
\begin{aligned}
& \left\|Q\left(x_{1}, y_{1}\right)-Q\left(x_{2}, y_{2}\right)\right\|_{Y} \\
& \quad=\left\|Q_{1}\left(x_{1}, y_{1}\right)-Q_{1}\left(x_{2}, y_{2}\right)\right\|+\left\|Q_{2}\left(x_{1}, y_{1}\right)-Q_{2}\left(x_{2}, y_{2}\right)\right\| \\
& \quad \leq\left[L_{1} M_{5}\left(M_{7}+M_{9}\right)+L_{2} M_{6}\left(M_{8}+M_{10}\right)\right]\left\|\left(x_{1}, y_{1}\right)-\left(x_{2}, y_{2}\right)\right\|_{Y} \\
& \quad=\Xi\left\|\left(x_{1}, y_{1}\right)-\left(x_{2}, y_{2}\right)\right\|_{Y} .
\end{aligned}
$$

By using the condition $\Xi<1$, we deduce that operator $Q$ is a contraction. By the Banach contraction mapping principle, we conclude that operator $Q$ has a unique fixed point $(x, y) \in \bar{B}_{r}$, which is the unique solution for problem $(S)-(B C)$ on $[0,1]$. 
(J3) The functions $f, g:[0,1] \times \mathbb{R}^{4} \rightarrow \mathbb{R}$ are continuous and there exist real constants $a_{i}, b_{i} \geq 0, i=0, \ldots, 4$, and at least one of $a_{0}$ and $b_{0}$ is positive, such that

$$
\left|f\left(t, u_{1}, u_{2}, u_{3}, u_{4}\right)\right| \leq a_{0}+\sum_{i=1}^{4} a_{i}\left|u_{i}\right|, \quad\left|g\left(t, u_{1}, u_{2}, u_{3}, u_{4}\right)\right| \leq b_{0}+\sum_{i=1}^{4} b_{i}\left|u_{i}\right|,
$$

for all $t \in[0,1], u_{i} \in \mathbb{R}, i=1, \ldots, 4$,

hold. If $\Xi_{1}:=\max \left\{M_{13}, M_{14}\right\}<1$, where $M_{13}=\left(a_{1}+\frac{a_{3}}{\Gamma\left(\theta_{1}+1\right)}\right)\left(M_{7}+M_{9}\right)+\left(b_{1}+\frac{b_{3}}{\Gamma\left(\theta_{2}+1\right)}\right)\left(M_{8}+\right.$ $\left.M_{10}\right)$ and $M_{14}=\left(a_{2}+\frac{a_{4}}{\Gamma\left(\sigma_{1}+1\right)}\right)\left(M_{7}+M_{9}\right)+\left(b_{2}+\frac{b_{4}}{\Gamma\left(\sigma_{2}+1\right)}\right)\left(M_{8}+M_{10}\right)$, then the boundary value problem $(S)-(B C)$ has at least one solution $(x(t), y(t)), t \in[0,1]$.

Proof We show that operator $Q$ is completely continuous. Because the functions $f$ and $g$ are continuous, we deduce that the operators $Q_{1}$ and $Q_{2}$ are continuous, and then $Q$ is a continuous operator. We will prove next that $Q$ is a compact operator, that is, it maps bounded sets into relatively compact sets. Let $\Omega \subset Y$ be a bounded set. Then there exist positive constants $L_{3}$ and $L_{4}$ such that $\left|\hat{f}_{x y}(t)\right| \leq L_{3}$ and $\left|\hat{g}_{x y}(t)\right| \leq L_{4}$ for all $t \in[0,1]$ and $(x, y) \in \Omega$. Hence we obtain as in the proof of Theorem 3.1 that

$$
\left|Q_{1}(x, y)(t)\right| \leq L_{3} M_{7}+L_{4} M_{10}, \quad\left|Q_{2}(x, y)(t)\right| \leq L_{3} M_{9}+L_{4} M_{8}
$$

for all $t \in[0,1]$ and $(x, y) \in \Omega$. So, we find

$$
\|Q(x, y)\|_{Y} \leq L_{3}\left(M_{7}+M_{9}\right)+L_{4}\left(M_{8}+M_{10}\right), \quad \forall(x, y) \in \Omega,
$$

and then $Q(\Omega)$ is uniformly bounded.

We show now that $Q(\Omega)$ are equicontinuous. Let $(x, y) \in \Omega$ and $t_{1}, t_{2} \in[0,1]$ with $t_{1}<t_{2}$. Then we have

$$
\begin{aligned}
&\left|Q_{1}(x, y)\left(t_{2}\right)-Q_{1}(x, y)\left(t_{1}\right)\right| \\
& \leq \mid-\frac{1}{\Gamma(\alpha)} \int_{0}^{t_{2}}\left(t_{2}-s\right)^{\alpha-1} \hat{f}_{x y}(s) d s+\frac{1}{\Gamma(\alpha)} \int_{0}^{t_{1}}\left(t_{1}-s\right)^{\alpha-1} \hat{f}_{x y}(s) d s \mid \\
&+\frac{\left(t_{2}^{\alpha-1}-t_{1}^{\alpha-1}\right) \Gamma(\beta)}{|\Delta| \Gamma\left(\alpha-\gamma_{0}\right) \Gamma\left(\beta-\delta_{0}\right)} \int_{0}^{1}(1-s)^{\alpha-\gamma_{0}-1}\left|\hat{f}_{x y}(s)\right| d s \\
&+\frac{\left(t_{2}^{\alpha-1}-t_{1}^{\alpha-1}\right) \Gamma(\beta)}{|\Delta| \Gamma\left(\beta-\delta_{0}\right)} \sum_{i=1}^{p} \frac{1}{\Gamma\left(\beta-\gamma_{i}\right)}\left|\int_{0}^{1}\left(\int_{0}^{s}(s-\tau)^{\beta-\gamma_{i}-1}\left|\hat{g}_{x y}(\tau)\right| d \tau\right) d H_{i}(s)\right| \\
&+\frac{t_{2}^{\alpha-1}-t_{1}^{\alpha-1}}{|\Delta|}\left(\sum_{i=1}^{p} \frac{\Gamma(\beta)}{\Gamma\left(\beta-\gamma_{i}\right)}\left|\int_{0}^{1} s^{\beta-\gamma_{i}-1} d H_{i}(s)\right|\right) \\
& \times\left(\frac{1}{\Gamma\left(\beta-\delta_{0}\right)} \int_{0}^{1}(1-s)^{\beta-\delta_{0}-1}\left|\hat{g}_{x y}(s)\right| d s\right) \\
&+\frac{t_{2}^{\alpha-1}-t_{1}^{\alpha-1}}{|\Delta|}\left(\sum_{i=1}^{p} \frac{\Gamma(\beta)}{\Gamma\left(\beta-\gamma_{i}\right)}\left|\int_{0}^{1} s^{\beta-\gamma_{i}-1} d H_{i}(s)\right|\right) \\
& \times\left(\sum_{i=1}^{q} \frac{1}{\Gamma\left(\alpha-\delta_{i}\right)}\left|\int_{0}^{1}\left(\int_{0}^{s}(s-\tau)^{\alpha-\delta_{i}-1}\left|\hat{f}_{x y}(\tau)\right| d \tau\right) d K_{i}(s)\right|\right)
\end{aligned}
$$




$$
\begin{aligned}
& \leq \frac{L_{3}}{\Gamma(\alpha)} \int_{0}^{t_{1}}\left[\left(t_{2}-s\right)^{\alpha-1}-\left(t_{1}-s\right)^{\alpha-1}\right] d s+\frac{L_{3}}{\Gamma(\alpha)} \int_{t_{1}}^{t_{2}}\left(t_{2}-s\right)^{\alpha-1} d s \\
& +\frac{L_{3}\left(t_{2}^{\alpha-1}-t_{1}^{\alpha-1}\right) \Gamma(\beta)}{|\Delta| \Gamma\left(\alpha-\gamma_{0}\right) \Gamma\left(\beta-\delta_{0}\right)} \int_{0}^{1}(1-s)^{\alpha-\gamma_{0}-1} d s \\
& +\frac{L_{4}\left(t_{2}^{\alpha-1}-t_{1}^{\alpha-1}\right) \Gamma(\beta)}{|\Delta| \Gamma\left(\beta-\delta_{0}\right)} \sum_{i=1}^{p} \frac{1}{\Gamma\left(\beta-\gamma_{i}\right)}\left|\int_{0}^{1}\left(\int_{0}^{s}(s-\tau)^{\beta-\gamma_{i}-1} d \tau\right) d H_{i}(s)\right| \\
& +\frac{L_{4}\left(t_{2}^{\alpha-1}-t_{1}^{\alpha-1}\right)}{|\Delta|}\left(\sum_{i=1}^{p} \frac{\Gamma(\beta)}{\Gamma\left(\beta-\gamma_{i}\right)}\left|\int_{0}^{1} s^{\beta-\gamma_{i}-1} d H_{i}(s)\right|\right) \\
& \times\left(\frac{1}{\Gamma\left(\beta-\delta_{0}\right)} \int_{0}^{1}(1-s)^{\beta-\delta_{0}-1} d s\right) \\
& +\frac{L_{3}\left(t_{2}^{\alpha-1}-t_{1}^{\alpha-1}\right)}{|\Delta|}\left(\sum_{i=1}^{p} \frac{\Gamma(\beta)}{\Gamma\left(\beta-\gamma_{i}\right)}\left|\int_{0}^{1} s^{\beta-\gamma_{i}-1} d H_{i}(s)\right|\right) \\
& \times\left(\sum_{i=1}^{q} \frac{1}{\Gamma\left(\alpha-\delta_{i}\right)}\left|\int_{0}^{1}\left(\int_{0}^{s}(s-\tau)^{\alpha-\delta_{i}-1} d \tau\right) d K_{i}(s)\right|\right) \\
& =\frac{L_{3}}{\Gamma(\alpha+1)}\left(t_{2}^{\alpha}-t_{1}^{\alpha}\right)+L_{3}\left(t_{2}^{\alpha-1}-t_{1}^{\alpha-1}\right)\left[\frac{\Gamma(\beta)}{|\Delta| \Gamma\left(\alpha-\gamma_{0}+1\right) \Gamma\left(\beta-\delta_{0}\right)}\right. \\
& \left.+\frac{1}{|\Delta|}\left(\sum_{i=1}^{p} \frac{\Gamma(\beta)}{\Gamma\left(\beta-\gamma_{i}\right)}\left|\int_{0}^{1} s^{\beta-\gamma_{i}-1} d H_{i}(s)\right|\right)\left(\sum_{i=1}^{q} \frac{1}{\Gamma\left(\alpha-\delta_{i}+1\right)}\left|\int_{0}^{1} s^{\alpha-\delta_{i}} d K_{i}(s)\right|\right)\right] \\
& +L_{4}\left(t_{2}^{\alpha-1}-t_{1}^{\alpha-1}\right)\left[\frac{\Gamma(\beta)}{|\Delta| \Gamma\left(\beta-\delta_{0}\right)} \sum_{i=1}^{p} \frac{1}{\Gamma\left(\beta-\gamma_{i}+1\right)}\left|\int_{0}^{1} s^{\beta-\gamma_{i}} d H_{i}(s)\right|\right. \\
& \left.+\frac{\Gamma(\beta)}{|\Delta| \Gamma\left(\beta-\delta_{0}+1\right)} \sum_{i=1}^{p} \frac{1}{\Gamma\left(\beta-\gamma_{i}\right)}\left|\int_{0}^{1} s^{\beta-\gamma_{i}-1} d H_{i}(s)\right|\right] \\
& =\frac{L_{3}}{\Gamma(\alpha+1)}\left(t_{2}^{\alpha}-t_{1}^{\alpha}\right)+\left(L_{3} M_{11}+L_{4} M_{10}\right)\left(t_{2}^{\alpha-1}-t_{1}^{\alpha-1}\right) \text {. }
\end{aligned}
$$

Hence we infer

$\left|Q_{1}(x, y)\left(t_{2}\right)-Q_{1}(x, y)\left(t_{1}\right)\right| \rightarrow 0, \quad$ as $t_{2} \rightarrow t_{1}$, uniformly with respect to $(x, y) \in \Omega$.

In a similar manner, for $(x, y) \in \Omega$ and $t_{1}, t_{2} \in[0,1]$ with $t_{1}<t_{2}$, we obtain

$$
\begin{aligned}
& \left|Q_{2}(x, y)\left(t_{2}\right)-Q_{2}(x, y)\left(t_{1}\right)\right| \\
& \leq \quad \frac{L_{4}}{\Gamma(\beta+1)}\left(t_{2}^{\beta}-t_{1}^{\beta}\right)+L_{4}\left(t_{2}^{\beta-1}-t_{1}^{\beta-1}\right)\left[\frac{\Gamma(\alpha)}{|\Delta| \Gamma\left(\alpha-\gamma_{0}\right) \Gamma\left(\beta-\delta_{0}+1\right)}\right. \\
& \left.\quad+\frac{1}{|\Delta|}\left(\sum_{i=1}^{q} \frac{\Gamma(\alpha)}{\Gamma\left(\alpha-\delta_{i}\right)}\left|\int_{0}^{1} s^{\alpha-\delta_{i}-1} d K_{i}(s)\right|\right)\left(\sum_{i=1}^{p} \frac{1}{\Gamma\left(\beta-\gamma_{i}+1\right)}\left|\int_{0}^{1} s^{\beta-\gamma_{i}} d H_{i}(s)\right|\right)\right] \\
& \quad+L_{3}\left(t_{2}^{\beta-1}-t_{1}^{\beta-1}\right)\left[\frac{\Gamma(\alpha)}{|\Delta| \Gamma\left(\alpha-\gamma_{0}\right)} \sum_{i=1}^{q} \frac{1}{\Gamma\left(\alpha-\delta_{i}+1\right)}\left|\int_{0}^{1} s^{\alpha-\delta_{i}} d K_{i}(s)\right|\right.
\end{aligned}
$$




$$
\begin{aligned}
& \left.+\frac{\Gamma(\alpha)}{|\Delta| \Gamma\left(\alpha-\gamma_{0}+1\right)} \sum_{i=1}^{q} \frac{1}{\Gamma\left(\alpha-\delta_{i}\right)}\left|\int_{0}^{1} s^{\alpha-\delta_{i}-1} d K_{i}(s)\right|\right] \\
= & \frac{L_{4}}{\Gamma(\beta+1)}\left(t_{2}^{\beta}-t_{1}^{\beta}\right)+\left(L_{4} M_{12}+L_{3} M_{9}\right)\left(t_{2}^{\beta-1}-t_{1}^{\beta-1}\right) .
\end{aligned}
$$

So we deduce

$$
\left|Q_{2}(x, y)\left(t_{2}\right)-Q_{2}(x, y)\left(t_{1}\right)\right| \rightarrow 0, \quad \text { as } t_{2} \rightarrow t_{1} \text {, uniformly with respect to }(x, y) \in \Omega \text {. }
$$

Then $Q_{1}(\Omega)$ and $Q_{2}(\Omega)$ are equicontinuous, and so $Q(\Omega)$ is also equicontinuous. Therefore, by Arzela-Ascoli theorem, we conclude that $Q(\Omega)$ is relatively compact, and then $Q$ is compact. We infer that operator $Q$ is completely continuous.

Next we will show that the set $U=\{(x, y) \in Y,(x, y)=v Q(x, y), 0<v<1\}$ is bounded. Let $(x, y) \in U$, that is, $(x, y)=v Q(x, y)$. Then for any $t \in[0,1]$, we get $x(t)=v Q_{1}(x, y)(t)$, $y(t)=v Q_{2}(x, y)(t)$. We denote the following functions:

$$
\begin{aligned}
& F_{x y}(s)=a_{0}+a_{1}|x(s)|+a_{2}|y(s)|+a_{3}\left|I_{0+}^{\theta_{1}} x(s)\right|+a_{4}\left|I_{0+}^{\sigma_{1}} y(s)\right|, \quad s \in[0,1], \\
& G_{x y}(s)=b_{0}+b_{1}|x(s)|+b_{2}|y(s)|+b_{3}\left|I_{0+}^{\theta_{2}} x(s)\right|+b_{4}\left|I_{0+}^{\sigma_{2}} y(s)\right|, \quad s \in[0,1] .
\end{aligned}
$$

By (J3), we find

$$
\begin{aligned}
|x(t)| \leq & \left|Q_{1}(x, y)(t)\right| \\
\leq & \frac{1}{\Gamma(\alpha)} \int_{0}^{t}(t-s)^{\alpha-1} F_{x y}(s) d s \\
& +\frac{t^{\alpha-1} \Gamma(\beta)}{|\Delta| \Gamma\left(\alpha-\gamma_{0}\right) \Gamma\left(\beta-\delta_{0}\right)} \int_{0}^{1}(1-s)^{\alpha-\gamma_{0}-1} F_{x y}(s) d s \\
& +\frac{t^{\alpha-1} \Gamma(\beta)}{|\Delta| \Gamma\left(\beta-\delta_{0}\right)} \sum_{i=1}^{p} \frac{1}{\Gamma\left(\beta-\gamma_{i}\right)}\left|\int_{0}^{1}\left(\int_{0}^{s}(s-\tau)^{\beta-\gamma_{i}-1} G_{x y}(\tau) d \tau\right) d H_{i}(s)\right| \\
& +\frac{t^{\alpha-1}}{|\Delta|}\left(\sum_{i=1}^{p} \frac{\Gamma(\beta)}{\Gamma\left(\beta-\gamma_{i}\right)}\left|\int_{0}^{1} s^{\beta-\gamma_{i}-1} d H_{i}(s)\right|\right) \\
& \times\left(\frac{1}{\Gamma\left(\beta-\delta_{0}\right)} \int_{0}^{1}(1-s)^{\beta-\delta_{0}-1} G_{x y}(s) d s\right) \\
& +\frac{t^{\alpha-1}}{|\Delta|}\left(\sum_{i=1}^{p} \frac{\Gamma(\beta)}{\Gamma\left(\beta-\gamma_{i}\right)}\left|\int_{0}^{1} s^{\beta-\gamma_{i}-1} d H_{i}(s)\right|\right) \\
& \times\left(\sum_{i=1}^{q} \frac{1}{\Gamma\left(\alpha-\delta_{i}\right)}\left|\int_{0}^{1}\left(\int_{0}^{s}(s-\tau)^{\alpha-\delta_{i}-1} F_{x y}(\tau) d \tau\right) d K_{i}(s)\right|\right) \\
\leq & \left(a_{0}+a_{1}\|x\|+a_{2}\|y\|+\frac{a_{3}}{\Gamma\left(\theta_{1}+1\right)}\|x\|+\frac{a_{4}}{\Gamma\left(\sigma_{1}+1\right)}\|y\|\right) \\
& \times\left[\frac{t^{\alpha}}{\Gamma(\alpha+1)}+\frac{t^{\alpha-1}}{|\Delta| \Gamma\left(\alpha-\gamma_{0}+1\right) \Gamma\left(\beta-\delta_{0}\right)}\left(\sum_{i=1}^{p} \frac{\Gamma(\beta)}{\Gamma\left(\beta-\gamma_{i}\right)}\left|\int_{0}^{1} s^{\beta-\gamma_{i}-1} d H_{i}(s)\right|\right)\right. \\
& {[\beta) }
\end{aligned}
$$




$$
\begin{aligned}
& \left.\times\left(\sum_{i=1}^{q} \frac{1}{\Gamma\left(\alpha-\delta_{i}+1\right)}\left|\int_{0}^{1} s^{\alpha-\delta_{i}} d K_{i}(s)\right|\right)\right] \\
& +\left(b_{0}+b_{1}\|x\|+b_{2}\|y\|+\frac{b_{3}}{\Gamma\left(\theta_{2}+1\right)}\|x\|+\frac{b_{4}}{\Gamma\left(\sigma_{2}+1\right)}\|y\|\right) \\
& \times\left[\frac{t^{\alpha-1} \Gamma(\beta)}{|\Delta| \Gamma\left(\beta-\delta_{0}\right)} \sum_{i=1}^{p} \frac{1}{\Gamma\left(\beta-\gamma_{i}+1\right)}\left|\int_{0}^{1} s^{\beta-\gamma_{i}} d H_{i}(s)\right|\right. \\
& \left.+\frac{t^{\alpha-1}}{|\Delta| \Gamma\left(\beta-\delta_{0}+1\right)} \sum_{i=1}^{p} \frac{\Gamma(\beta)}{\Gamma\left(\beta-\gamma_{i}\right)}\left|\int_{0}^{1} s^{\beta-\gamma_{i}-1} d H_{i}(s)\right|\right], \quad \forall t \in[0,1] .
\end{aligned}
$$

Therefore we deduce

$$
\begin{aligned}
\|x\| \leq & \left(a_{0}+a_{1}\|x\|+a_{2}\|y\|+\frac{a_{3}}{\Gamma\left(\theta_{1}+1\right)}\|x\|+\frac{a_{4}}{\Gamma\left(\sigma_{1}+1\right)}\|y\|\right) M_{7} \\
& +\left(b_{0}+b_{1}\|x\|+b_{2}\|y\|+\frac{b_{3}}{\Gamma\left(\theta_{2}+1\right)}\|x\|+\frac{b_{4}}{\Gamma\left(\sigma_{2}+1\right)}\|y\|\right) M_{10} .
\end{aligned}
$$

In a similar manner, we obtain

$$
\begin{aligned}
\|y\| \leq & \left(a_{0}+a_{1}\|x\|+a_{2}\|y\|+\frac{a_{3}}{\Gamma\left(\theta_{1}+1\right)}\|x\|+\frac{a_{4}}{\Gamma\left(\sigma_{1}+1\right)}\|y\|\right) M_{9} \\
& +\left(b_{0}+b_{1}\|x\|+b_{2}\|y\|+\frac{b_{3}}{\Gamma\left(\theta_{2}+1\right)}\|x\|+\frac{b_{4}}{\Gamma\left(\sigma_{2}+1\right)}\|y\|\right) M_{8} .
\end{aligned}
$$

By (10) and (11), we infer

$$
\begin{aligned}
\|(x, y) & \|_{Y} \\
= & \|x\|+\|y\| \leq a_{0}\left(M_{7}+M_{9}\right)+b_{0}\left(M_{8}+M_{10}\right) \\
& +\left[a_{1}\left(M_{7}+M_{9}\right)+\frac{a_{3}}{\Gamma\left(\theta_{1}+1\right)}\left(M_{7}+M_{9}\right)+b_{1}\left(M_{8}+M_{10}\right)\right. \\
& \left.+\frac{b_{3}}{\Gamma\left(\theta_{2}+1\right)}\left(M_{8}+M_{10}\right)\right]\|x\| \\
& +\left[a_{2}\left(M_{7}+M_{9}\right)+\frac{a_{4}}{\Gamma\left(\sigma_{1}+1\right)}\left(M_{7}+M_{9}\right)+b_{2}\left(M_{8}+M_{10}\right)\right. \\
& \left.+\frac{b_{4}}{\Gamma\left(\theta_{2}+1\right)}\left(M_{8}+M_{10}\right)\right]\|y\| \\
= & a_{0}\left(M_{7}+M_{9}\right)+b_{0}\left(M_{8}+M_{10}\right)+M_{13}\|x\|+M_{14}\|y\| \\
\leq & a_{0}\left(M_{7}+M_{9}\right)+b_{0}\left(M_{8}+M_{10}\right)+\Xi_{1}\|(x, y)\|_{Y} .
\end{aligned}
$$

Because $\Xi_{1}<1$, we find

$$
\|(x, y)\|_{Y} \leq\left[a_{0}\left(M_{7}+M_{9}\right)+b_{0}\left(M_{8}+M_{10}\right)\right]\left(1-\Xi_{1}\right)^{-1}, \quad \forall(x, y) \in U
$$

So we deduce that the set $U$ is bounded.

By using the Leray-Schauder alternative theorem, we conclude that operator $Q$ has at least one fixed point, which is a solution for our problem $(S)-(B C)$. 
Theorem 3.3 Assume that (J1), (J2), and

(J4) There exist the functions $\psi_{1}, \psi_{2} \in C([0,1],[0, \infty))$ such that

$$
\left|f\left(t, u_{1}, u_{2}, u_{3}, u_{4}\right)\right| \leq \psi_{1}(t), \quad\left|g\left(t, u_{1}, u_{2}, u_{3}, u_{4}\right)\right| \leq \psi_{2}(t)
$$

for all $t \in[0,1], u_{i} \in \mathbb{R}, i=1, \ldots, 4$,

hold. If $\Xi_{2}:=L_{1} M_{5} \frac{1}{\Gamma(\alpha+1)}+L_{2} M_{6} \frac{1}{\Gamma(\beta+1)}<1$, then problem $(S)-(B C)$ has at least one solution on $[0,1]$.

Proof We fix $r_{1}>0$ such that $r_{1} \geq\left(M_{7}+M_{9}\right)\left\|\psi_{1}\right\|+\left(M_{8}+M_{10}\right)\left\|\psi_{2}\right\|$. We consider the set $\bar{B}_{r_{1}}=\left\{(x, y) \in Y,\|(x, y)\|_{Y} \leq r_{1}\right\}$, and introduce the operators $D=\left(D_{1}, D_{2}\right): \bar{B}_{r_{1}} \rightarrow Y$ and $E=\left(E_{1}, E_{2}\right): \bar{B}_{r_{1}} \rightarrow Y$, where $D_{1}, D_{2}, E_{1}, E_{2}: \bar{B}_{r_{1}} \rightarrow X$ are defined by

$$
\begin{aligned}
& D_{1}(x, y)(t)=\frac{1}{\Gamma(\alpha)} \int_{0}^{t}(t-s)^{\alpha-1} \hat{f}_{x y}(s) d s, \\
& E_{1}(x, y)(t)=\frac{t^{\alpha-1} \Gamma(\beta)}{\Delta \Gamma\left(\alpha-\gamma_{0}\right) \Gamma\left(\beta-\delta_{0}\right)} \int_{0}^{1}(1-s)^{\alpha-\gamma_{0}-1} \hat{f}_{x y}(s) d s \\
& -\frac{t^{\alpha-1} \Gamma(\beta)}{\Delta \Gamma\left(\beta-\delta_{0}\right)} \sum_{i=1}^{p} \frac{1}{\Gamma\left(\beta-\gamma_{i}\right)} \int_{0}^{1}\left(\int_{0}^{s}(s-\tau)^{\beta-\gamma_{i}-1} \hat{g}_{x y}(\tau) d \tau\right) d H_{i}(s) \\
& +\frac{t^{\alpha-1} \Delta_{1}}{\Delta \Gamma\left(\beta-\delta_{0}\right)} \int_{0}^{1}(1-s)^{\beta-\delta_{0}-1} \hat{g}_{x y}(s) d s \\
& -\frac{t^{\alpha-1} \Delta_{1}}{\Delta}\left(\sum_{i=1}^{q} \frac{1}{\Gamma\left(\alpha-\delta_{i}\right)} \int_{0}^{1}\left(\int_{0}^{s}(s-\tau)^{\alpha-\delta_{i}-1} \hat{f}_{x y}(\tau) d \tau\right) d K_{i}(s)\right) \\
& D_{2}(x, y)(t)=-\frac{1}{\Gamma(\beta)} \int_{0}^{t}(t-s)^{\beta-1} \hat{g}_{x y}(s) d s \text {, } \\
& E_{2}(x, y)(t)=\frac{t^{\beta-1} \Gamma(\alpha)}{\Delta \Gamma\left(\alpha-\gamma_{0}\right) \Gamma\left(\beta-\delta_{0}\right)} \int_{0}^{1}(1-s)^{\beta-\delta_{0}-1} \hat{g}_{x y}(s) d s \\
& -\frac{t^{\beta-1} \Gamma(\alpha)}{\Delta \Gamma\left(\alpha-\gamma_{0}\right)} \sum_{i=1}^{q} \frac{1}{\Gamma\left(\alpha-\delta_{i}\right)} \int_{0}^{1}\left(\int_{0}^{s}(s-\tau)^{\alpha-\delta_{i}-1} \hat{f}_{x y}(\tau) d \tau\right) d K_{i}(s) \\
& +\frac{t^{\beta-1} \Delta_{2}}{\Delta \Gamma\left(\alpha-\gamma_{0}\right)} \int_{0}^{1}(1-s)^{\alpha-\gamma_{0}-1} \hat{f}_{x y}(s) d s \\
& -\frac{t^{\beta-1} \Delta_{2}}{\Delta}\left(\sum_{i=1}^{p} \frac{1}{\Gamma\left(\beta-\gamma_{i}\right)} \int_{0}^{1}\left(\int_{0}^{s}(s-\tau)^{\beta-\gamma_{i}-1} \hat{g}_{x y}(\tau) d \tau\right) d H_{i}(s)\right),
\end{aligned}
$$

for all $t \in[0,1]$ and $(x, y) \in \bar{B}_{r_{1}}$. So $Q_{1}=D_{1}+E_{1}, Q_{2}=D_{2}+E_{2}$, and $Q=D+E$.

By using (J4), we find for all $\left(x_{1}, y_{1}\right),\left(x_{2}, y_{2}\right) \in \bar{B}_{r_{1}}$ as in the proof of Theorem 3.1 that

$$
\begin{aligned}
& \left\|D\left(x_{1}, y_{1}\right)+E\left(x_{2}, y_{2}\right)\right\|_{Y} \\
& \quad \leq\left\|D\left(x_{1}, y_{1}\right)\right\|_{Y}+\left\|E\left(x_{2}, y_{2}\right)\right\|_{Y} \\
& \quad=\left\|D_{1}\left(x_{1}, y_{1}\right)\right\|+\left\|D_{2}\left(x_{1}, y_{1}\right)\right\|+\left\|E_{1}\left(x_{2}, y_{2}\right)\right\|+\left\|E_{2}\left(x_{2}, y_{2}\right)\right\| \\
& \quad \leq \frac{1}{\Gamma(\alpha+1)}\left\|\psi_{1}\right\|+\frac{1}{\Gamma(\beta+1)}\left\|\psi_{2}\right\|+\left(M_{11}\left\|\psi_{1}\right\|+M_{10}\left\|\psi_{2}\right\|\right)
\end{aligned}
$$




$$
\begin{aligned}
& +\left(M_{9}\left\|\psi_{1}\right\|+M_{12}\left\|\psi_{2}\right\|\right) \\
= & \left(M_{7}+M_{9}\right)\left\|\psi_{1}\right\|+\left(M_{8}+M_{10}\right)\left\|\psi_{2}\right\| \leq r_{1} .
\end{aligned}
$$

So $D\left(x_{1}, y_{1}\right)+E\left(x_{2}, y_{2}\right) \in \bar{B}_{r_{1}}$ for all $\left(x_{1}, y_{1}\right),\left(x_{2}, y_{2}\right) \in \bar{B}_{r_{1}}$.

The operator $D$ is a contraction because

$$
\begin{aligned}
& \left\|D\left(x_{1}, y_{1}\right)-D\left(x_{2}, y_{2}\right)\right\|_{Y} \\
& \quad=\left\|D_{1}\left(x_{1}, y_{1}\right)-D_{1}\left(x_{2}, y_{2}\right)\right\|+\left\|D_{2}\left(x_{1}, y_{1}\right)-D_{2}\left(x_{2}, y_{2}\right)\right\| \\
& \quad \leq\left(L_{1} M_{5} \frac{1}{\Gamma(\alpha+1)}+L_{2} M_{6} \frac{1}{\Gamma(\beta+1)}\right)\left(\left\|x_{1}-x_{2}\right\|+\left\|y_{1}-y_{2}\right\|\right) \\
& \quad=\Xi_{2}\left\|\left(x_{1}, y_{1}\right)-\left(x_{2}, y_{2}\right)\right\|_{Y},
\end{aligned}
$$

for all $\left(x_{1}, y_{1}\right),\left(x_{2}, y_{2}\right) \in \bar{B}_{r_{1}}$, and $\Xi_{2}<1$.

Because the functions $f$ and $g$ are continuous, we obtain that operator $E$ is continuous on $\bar{B}_{r_{1}}$. We show next that $E$ is compact. The functions from $E$ are uniformly bounded on $\bar{B}_{r_{1}}$ because

$$
\begin{aligned}
& \|E(x, y)\|_{Y}=\left\|E_{1}(x, y)\right\|+\left\|E_{2}(x, y)\right\| \leq\left(M_{11}+M_{9}\right)\left\|\psi_{1}\right\|+\left(M_{10}+M_{12}\right)\left\|\psi_{2}\right\|, \\
& \forall(x, y) \in \bar{B}_{r_{1}} .
\end{aligned}
$$

We prove next that the functions from $E\left(\bar{B}_{r_{1}}\right)$ are equicontinuous. We denote by

$$
\begin{aligned}
\Psi_{r_{1}}= & \sup \left\{|f(t, x, y, u, v)|, t \in[0,1],|x| \leq r_{1},|y| \leq r_{1},|u| \leq \frac{r_{1}}{\Gamma\left(\theta_{1}+1\right)},\right. \\
& \left.|v| \leq \frac{r_{1}}{\Gamma\left(\sigma_{1}+1\right)}\right\}, \\
\Theta_{r_{1}}= & \sup \left\{|g(t, x, y, u, v)|, t \in[0,1],|x| \leq r_{1},|y| \leq r_{1},|u| \leq \frac{r_{1}}{\Gamma\left(\theta_{2}+1\right)},\right. \\
& \left.|v| \leq \frac{r_{1}}{\Gamma\left(\sigma_{2}+1\right)}\right\} .
\end{aligned}
$$

Then for $(x, y) \in \bar{B}_{r_{1}}$ and $t_{1}, t_{2} \in[0,1]$ with $t_{1}<t_{2}$, we deduce

$$
\begin{aligned}
& \left|E_{1}(x, y)\left(t_{2}\right)-E_{1}(x, y)\left(t_{1}\right)\right| \\
& \leq \frac{\left(t_{2}^{\alpha-1}-t_{1}^{\alpha-1}\right) \Gamma(\beta)}{|\Delta| \Gamma\left(\alpha-\gamma_{0}\right) \Gamma\left(\beta-\delta_{0}\right)} \int_{0}^{1}(1-s)^{\alpha-\gamma_{0}-1} \Psi_{r_{1}} d s \\
& \quad+\frac{\left(t_{2}^{\alpha-1}-t_{1}^{\alpha-1}\right) \Gamma(\beta)}{|\Delta| \Gamma\left(\beta-\delta_{0}\right)} \sum_{i=1}^{p} \frac{1}{\Gamma\left(\beta-\gamma_{i}\right)}\left|\int_{0}^{1}\left(\int_{0}^{s}(s-\tau)^{\beta-\gamma_{i}-1} \Theta_{r_{1}} d \tau\right) d H_{i}(s)\right| \\
& \quad+\frac{t_{2}^{\alpha-1}-t_{1}^{\alpha-1}}{|\Delta|}\left(\sum_{i=1}^{p} \frac{\Gamma(\beta)}{\Gamma\left(\beta-\gamma_{i}\right)}\left|\int_{0}^{1} s^{\beta-\gamma_{i}-1} d H_{i}(s)\right|\right) \\
& \quad \times\left(\frac{1}{\Gamma\left(\beta-\delta_{0}\right)} \int_{0}^{1}(1-s)^{\beta-\delta_{0}-1} \Theta_{r_{1}} d s\right)
\end{aligned}
$$


Luck Advances in Difference Equations

(2021) 2021:134

Page 17 of 25

$$
\begin{aligned}
& +\frac{t_{2}^{\alpha-1}-t_{1}^{\alpha-1}}{|\Delta|}\left(\sum_{i=1}^{p} \frac{\Gamma(\beta)}{\Gamma\left(\beta-\gamma_{i}\right)}\left|\int_{0}^{1} s^{\beta-\gamma_{i}-1} d H_{i}(s)\right|\right) \\
& \times\left(\sum_{i=1}^{q} \frac{1}{\Gamma\left(\alpha-\delta_{i}\right)}\left|\int_{0}^{1}\left(\int_{0}^{s}(s-\tau)^{\alpha-\delta_{i}-1} \Psi_{r_{1}} d \tau\right) d K_{i}(s)\right|\right) \\
= & \Psi_{r_{1}}\left(t_{2}^{\alpha-1}-t_{1}^{\alpha-1}\right)\left[\frac{\Gamma(\beta)}{|\Delta| \Gamma\left(\alpha-\gamma_{0}+1\right) \Gamma\left(\beta-\delta_{0}\right)}\right. \\
& \left.+\frac{1}{|\Delta|}\left(\sum_{i=1}^{p} \frac{\Gamma(\beta)}{\Gamma\left(\beta-\gamma_{i}\right)}\left|\int_{0}^{1} s^{\beta-\gamma_{i}-1} d H_{i}(s)\right|\right)\left(\sum_{i=1}^{p} \frac{1}{\Gamma\left(\alpha-\delta_{i}+1\right)}\left|\int_{0}^{1} s^{\alpha-\delta_{i}} d K_{i}(s)\right|\right)\right] \\
& +\Theta_{r_{1}}\left(t_{2}^{\alpha-1}-t_{1}^{\alpha-1}\right)\left[\frac{\Gamma(\beta)}{|\Delta| \Gamma\left(\beta-\delta_{0}\right)} \sum_{i=1}^{p} \frac{1}{\Gamma\left(\beta-\gamma_{i}+1\right)}\left|\int_{0}^{1} s^{\beta-\gamma_{i}} d H_{i}(s)\right|\right. \\
& \left.+\frac{1}{|\Delta| \Gamma\left(\beta-\delta_{0}+1\right)} \sum_{i=1}^{p} \frac{\Gamma(\beta)}{\Gamma\left(\beta-\gamma_{i}\right)}\left|\int_{0}^{1} s^{\beta-\gamma_{i}-1} d H_{i}(s)\right|\right] \\
= & M_{11} \Psi_{r_{1}}\left(t_{2}^{\alpha-1}-t_{1}^{\alpha-1}\right)+M_{10} \Theta_{r_{1}}\left(t_{2}^{\alpha-1}-t_{1}^{\alpha-1}\right),
\end{aligned}
$$

$$
\begin{aligned}
& \left|E_{2}(x, y)\left(t_{2}\right)-E_{2}(x, y)\left(t_{1}\right)\right| \\
& \leq \frac{\left(t_{2}^{\beta-1}-t_{1}^{\beta-1}\right) \Gamma(\alpha)}{|\Delta| \Gamma\left(\alpha-\gamma_{0}\right) \Gamma\left(\beta-\delta_{0}\right)} \int_{0}^{1}(1-s)^{\beta-\delta_{0}-1} \Theta_{r_{1}} d s \\
& +\frac{\left(t_{2}^{\beta-1}-t_{1}^{\beta-1}\right) \Gamma(\alpha)}{|\Delta| \Gamma\left(\alpha-\gamma_{0}\right)} \sum_{i=1}^{q} \frac{1}{\Gamma\left(\alpha-\delta_{i}\right)}\left|\int_{0}^{1}\left(\int_{0}^{s}(s-\tau)^{\alpha-\delta_{i}-1} \Psi_{r_{1}} d \tau\right) d K_{i}(s)\right| \\
& +\frac{t_{2}^{\beta-1}-t_{1}^{\beta-1}}{|\Delta|}\left(\sum_{i=1}^{q} \frac{\Gamma(\alpha)}{\Gamma\left(\alpha-\delta_{i}\right)}\left|\int_{0}^{1} s^{\alpha-\delta_{i}-1} d K_{i}(s)\right|\right) \\
& \times\left(\frac{1}{\Gamma\left(\alpha-\gamma_{0}\right)} \int_{0}^{1}(1-s)^{\alpha-\gamma_{0}-1} \Psi_{r_{1}} d s\right) \\
& +\frac{t_{2}^{\beta-1}-t_{1}^{\beta-1}}{|\Delta|}\left(\sum_{i=1}^{q} \frac{\Gamma(\alpha)}{\Gamma\left(\alpha-\delta_{i}\right)}\left|\int_{0}^{1} s^{\alpha-\delta_{i}-1} d K_{i}(s)\right|\right) \\
& \times\left(\sum_{i=1}^{p} \frac{1}{\Gamma\left(\beta-\gamma_{i}\right)}\left|\int_{0}^{1}\left(\int_{0}^{s}(s-\tau)^{\beta-\gamma_{i}-1} \Theta_{r_{1}} d \tau\right) d H_{i}(s)\right|\right) \\
& =\Psi_{r_{1}}\left(t_{2}^{\beta-1}-t_{1}^{\beta-1}\right)\left[\frac{\Gamma(\alpha)}{|\Delta| \Gamma\left(\alpha-\gamma_{0}\right)} \sum_{i=1}^{q} \frac{1}{\Gamma\left(\alpha-\delta_{i}+1\right)}\left|\int_{0}^{1} s^{\alpha-\delta_{i}} d K_{i}(s)\right|\right. \\
& \left.+\frac{1}{|\Delta| \Gamma\left(\alpha-\gamma_{0}+1\right)} \sum_{i=1}^{q} \frac{\Gamma(\alpha)}{\Gamma\left(\alpha-\delta_{i}\right)}\left|\int_{0}^{1} s^{\alpha-\delta_{i}-1} d K_{i}(s)\right|\right] \\
& +\Theta_{r_{1}}\left(t_{2}^{\beta-1}-t_{1}^{\beta-1}\right)\left[\frac{\Gamma(\alpha)}{|\Delta| \Gamma\left(\alpha-\gamma_{0}\right) \Gamma\left(\beta-\delta_{0}+1\right)}\right. \\
& \left.+\frac{1}{|\Delta|}\left(\sum_{i=1}^{q} \frac{\Gamma(\alpha)}{\Gamma\left(\alpha-\delta_{i}\right)}\left|\int_{0}^{1} s^{\alpha-\delta_{i}-1} d K_{i}(s)\right|\right)\left(\sum_{i=1}^{p} \frac{1}{\Gamma\left(\beta-\gamma_{i}+1\right)}\left|\int_{0}^{1} s^{\beta-\gamma_{i}} d H_{i}(s)\right|\right)\right] \\
& =M_{9} \Psi_{r_{1}}\left(t_{2}^{\beta-1}-t_{1}^{\beta-1}\right)+M_{12} \Theta_{r_{1}}\left(t_{2}^{\beta-1}-t_{1}^{\beta-1}\right) \text {. }
\end{aligned}
$$


Therefore we infer

$$
\left|E_{1}(x, y)\left(t_{2}\right)-E_{1}(x, y)\left(t_{1}\right)\right| \rightarrow 0, \quad\left|E_{2}(x, y)\left(t_{2}\right)-E_{2}(x, y)\left(t_{1}\right)\right| \rightarrow 0,
$$

as $t_{2} \rightarrow t_{1}$, uniformly with respect to $(x, y) \in \bar{B}_{r_{1}}$. Then $E_{1}\left(\bar{B}_{r_{1}}\right)$ and $E_{2}\left(\bar{B}_{r_{1}}\right)$ are equicontinuous, and so $E\left(\bar{B}_{r_{1}}\right)$ is also equicontinuous. By applying Arzela-Ascoli theorem, we conclude that the set $E\left(\bar{B}_{r_{1}}\right)$ is relatively compact. Hence $E$ is a compact operator on $\bar{B}_{r_{1}}$. By using the Krasnosel'skii theorem for the sum of two operators (see [22]), we deduce that there exists a fixed point of operator $D+E(=Q)$, which is a solution of problem $(S)-(B C)$.

Theorem 3.4 Suppose that (J1), (J2), and (J4) hold. If $\Xi_{3}:=L_{1} M_{5}\left(M_{9}+M_{11}\right)+L_{2} M_{6}\left(M_{10}+\right.$ $\left.M_{12}\right)<1$, then problem $(S)-(B C)$ has at least one solution $(x, y)$ on $[0,1]$.

Proof We consider again a positive number $r_{1} \geq\left(M_{7}+M_{9}\right)\left\|\psi_{1}\right\|+\left(M_{8}+M_{10}\right)\left\|\psi_{2}\right\|$ and the operators $D$ and $E$ defined on $\bar{B}_{r_{1}}$ given by (12). As in the proof of Theorem 3.3, we have $D\left(x_{1}, y_{1}\right)+E\left(x_{2}, y_{2}\right) \in \bar{B}_{r_{1}}$ for all $\left(x_{1}, y_{1}\right),\left(x_{2}, y_{2}\right) \in \bar{B}_{r_{1}}$.

The operator $E$ is a contraction because

$$
\begin{aligned}
&\left\|E\left(x_{1}, y_{1}\right)-E\left(x_{2}, y_{2}\right)\right\|_{Y} \\
&=\left\|E_{1}\left(x_{1}, y_{1}\right)-E_{1}\left(x_{1}, y_{1}\right)\right\|+\left\|E_{2}\left(x_{1}, y_{1}\right)-E_{2}\left(x_{2}, y_{2}\right)\right\| \\
& \leq\left(L_{1} M_{5} M_{11}+L_{2} M_{6} M_{10}\right)\left\|\left(x_{1}, y_{1}\right)-\left(x_{2}, y_{2}\right)\right\|_{Y} \\
& \quad+\left(L_{1} M_{5} M_{9}+L_{2} M_{6} M_{12}\right)\left\|\left(x_{1}, y_{1}\right)-\left(x_{2}, y_{2}\right)\right\|_{Y} \\
&=\left(L_{1} M_{5}\left(M_{9}+M_{11}\right)+L_{2} M_{6}\left(M_{10}+M_{12}\right)\right)\left\|\left(x_{1}, y_{1}\right)-\left(x_{2}, y_{2}\right)\right\|_{Y} \\
&= \Xi_{3}\left\|\left(x_{1}, y_{1}\right)-\left(x_{2}, y_{2}\right)\right\|_{Y},
\end{aligned}
$$

for all $\left(x_{1}, y_{1}\right),\left(x_{2}, y_{2}\right) \in \bar{B}_{r_{1}}$, with $\Xi_{3}<1$.

In what follows, the continuity of functions $f$ and $g$ implies that operator $D$ is continuous on $\bar{B}_{r_{1}}$. We prove now that $D$ is a compact operator. The functions from $D\left(\bar{B}_{r_{1}}\right)$ are uniformly bounded because

$$
\|D(x, y)\|_{Y}=\left\|D_{1}(x, y)\right\|+\left\|D_{2}(x, y)\right\| \leq \frac{1}{\Gamma(\alpha+1)}\left\|\psi_{1}\right\|+\frac{1}{\Gamma(\beta+1)}\left\|\psi_{2}\right\|, \quad \forall(x, y) \in \bar{B}_{r_{1}} .
$$

Now we show that the functions from $D\left(\bar{B}_{r_{1}}\right)$ are equicontinuous. By using $\Psi_{r_{1}}$ and $\Theta_{r_{1}}$ defined by (13), we deduce that for $(x, y) \in \bar{B}_{r_{1}}$ and $t_{1}, t_{2} \in[0,1]$ with $t_{1}<t_{2}$ that

$$
\begin{aligned}
& \left|D_{1}(x, y)\left(t_{2}\right)-D_{1}(x, y)\left(t_{1}\right)\right| \leq \frac{\Psi_{r_{1}}}{\Gamma(\alpha+1)}\left(t_{2}^{\alpha}-t_{1}^{\alpha}\right), \\
& \left|D_{2}(x, y)\left(t_{2}\right)-D_{2}(x, y)\left(t_{1}\right)\right| \leq \frac{\Theta_{r_{1}}}{\Gamma(\beta+1)}\left(t_{2}^{\beta}-t_{1}^{\beta}\right) .
\end{aligned}
$$

Therefore we conclude

$$
\left|D_{1}(x, y)\left(t_{2}\right)-D_{1}(x, y)\left(t_{1}\right)\right| \rightarrow 0, \quad\left|D_{2}(x, y)\left(t_{2}\right)-D_{2}(x, y)\left(t_{1}\right)\right| \rightarrow 0,
$$


as $t_{2} \rightarrow t_{1}$, uniformly with respect to $(x, y) \in \bar{B}_{r_{1}}$. We infer that $D_{1}\left(\bar{B}_{r_{1}}\right)$ and $D_{2}\left(\bar{B}_{r_{1}}\right)$ are equicontinuous, and so $D\left(\bar{B}_{r_{1}}\right)$ is equicontinuous. By using Arzela-Ascoli theorem, we deduce that the set $D\left(\bar{B}_{r_{1}}\right)$ is relatively compact. Then $D$ is a compact operator on $\bar{B}_{r_{1}}$. By using the Krasnosel'skii theorem, we conclude that there exists a fixed point of operator $D+E(=Q)$, which is a solution of problem $(S)-(B C)$.

Theorem 3.5 Assume that (J1) and

(J5) The functions $f, g:[0,1] \times \mathbb{R}^{4} \rightarrow \mathbb{R}$ are continuous and there exist the constants $c_{i} \geq$ $0, i=0, \ldots, 4$ with at least one nonzero constant, the constants $d_{i} \geq 0, i=0, \ldots, 4$ with at least one nonzero constant, and $l_{i}, m_{i} \in(0,1), i=1, \ldots, 4$ such that

$$
\begin{aligned}
& \left|f\left(t, u_{1}, u_{2}, u_{3}, u_{4}\right)\right| \leq c_{0}+\sum_{i=1}^{4} c_{i}\left|u_{i}\right|^{l_{i},} \\
& \left|g\left(t, u_{1}, u_{2}, u_{3}, u_{4}\right)\right| \leq d_{0}+\sum_{i=1}^{4} d_{i}\left|u_{i}\right|^{m_{i}},
\end{aligned}
$$

for all $t \in[0,1], u_{i} \in \mathbb{R}, i=1, \ldots, 4$,

hold. Then problem $(S)-(B C)$ has at least one solution.

Proof Let $\bar{B}_{R}=\left\{(x, y) \in Y,\|(x, y)\|_{Y} \leq R\right\}$, where

$$
\begin{aligned}
R \geq & \max \left\{20 c_{0} M_{7},\left(20 c_{1} M_{7}\right)^{\frac{1}{1-l_{1}}},\left(20 c_{2} M_{7}\right)^{\frac{1}{1-l_{2}}},\right. \\
& \left(\frac{20 c_{3} M_{7}}{\left(\Gamma\left(\theta_{1}+1\right)\right)^{l_{3}}}\right)^{\frac{1}{1-l_{3}}},\left(\frac{20 c_{4} M_{7}}{\left(\Gamma\left(\sigma_{1}+1\right)\right)^{l_{4}}}\right)^{\frac{1}{1-l_{4}}}, \\
& 20 d_{0} M_{10},\left(20 d_{1} M_{10}\right)^{\frac{1}{1-m_{1}}},\left(20 d_{2} M_{10}\right)^{\frac{1}{1-m_{2}}}, \\
& \left(\frac{20 d_{3} M_{10}}{\left(\Gamma\left(\theta_{2}+1\right)\right)^{m_{3}}}\right)^{\frac{1}{1-m_{3}}},\left(\frac{20 d_{4} M_{10}}{\left(\Gamma\left(\sigma_{2}+1\right)\right)^{m_{4}}}\right)^{\frac{1}{1-m_{4}}}, \\
& 20 c_{0} M_{9},\left(20 c_{1} M_{9}\right)^{\frac{1}{1-l_{1}}},\left(20 c_{2} M_{9}\right)^{\frac{1}{1-l_{2}}}, \\
& \left(\frac{20 c_{3} M_{9}}{\left(\Gamma\left(\theta_{1}+1\right)\right)^{l_{3}}}\right)^{\frac{1}{1-l_{3}}},\left(\frac{20 c_{4} M_{9}}{\left(\Gamma\left(\sigma_{1}+1\right)\right)^{l_{4}}}\right)^{\frac{1}{1-l_{4}}}, \\
& 20 d_{0} M_{8},\left(20 d_{1} M_{8}\right)^{\frac{1}{1-m_{1}}},\left(20 d_{2} M_{8}\right)^{\frac{1}{1-m_{2}}}, \\
& \left.\left(\frac{20 d_{3} M_{8}}{\left(\Gamma\left(\theta_{2}+1\right)\right)^{m_{3}}}\right)^{\frac{1}{1-m_{3}}},\left(\frac{20 d_{4} M_{8}}{\left(\Gamma\left(\sigma_{2}+1\right)\right)^{m_{4}}}\right)^{\frac{1}{1-m_{4}}}\right\} .
\end{aligned}
$$

We prove that $Q: \bar{B}_{R} \rightarrow \bar{B}_{R}$. For $(x, y) \in \bar{B}_{R}$, we have

$$
\begin{aligned}
\left|Q_{1}(x, y)(t)\right| \leq & \left(c_{0}+c_{1} R^{l_{1}}+c_{2} R^{l_{2}}+c_{3} \frac{R^{l_{3}}}{\left(\Gamma\left(\theta_{1}+1\right)\right)^{l_{3}}}+c_{4} \frac{R^{l_{4}}}{\left(\Gamma\left(\sigma_{1}+1\right)\right)^{l_{4}}}\right) M_{7} \\
& +\left(d_{0}+d_{1} R^{m_{1}}+d_{2} R^{m_{2}}+d_{3} \frac{R^{m_{3}}}{\left(\Gamma\left(\theta_{2}+1\right)\right)^{m_{3}}}+d_{4} \frac{R^{m_{4}}}{\left(\Gamma\left(\sigma_{2}+1\right)\right)^{m_{4}}}\right) M_{10} \\
\leq & \frac{R}{2}
\end{aligned}
$$




$$
\begin{aligned}
\left|Q_{2}(x, y)(t)\right| \leq & \left(c_{0}+c_{1} R^{l_{1}}+c_{2} R^{l_{2}}+c_{3} \frac{R^{l_{3}}}{\left(\Gamma\left(\theta_{1}+1\right)\right)^{l_{3}}}+c_{4} \frac{R^{l_{4}}}{\left(\Gamma\left(\sigma_{1}+1\right)\right)^{l_{4}}}\right) M_{9} \\
& +\left(d_{0}+d_{1} R^{m_{1}}+d_{2} R^{m_{2}}+d_{3} \frac{R^{m_{3}}}{\left(\Gamma\left(\theta_{2}+1\right)\right)^{m_{3}}}+d_{4} \frac{R^{m_{4}}}{\left(\Gamma\left(\sigma_{2}+1\right)\right)^{m_{4}}}\right) M_{8} \\
\leq & \frac{R}{2}
\end{aligned}
$$

for all $t \in[0,1]$. Then we obtain

$$
\| Q(x, y))\left\|_{Y}=\right\| Q_{1}(x, y)\|+\| Q_{2}(x, y) \| \leq R, \quad \forall(x, y) \in \bar{B}_{R},
$$

which implies that $Q\left(\bar{B}_{R}\right) \subset \bar{B}_{R}$.

By using the fact that the functions $f$ and $g$ are continuous, we deduce that that operator $Q$ is continuous on $\bar{B}_{R}$. Besides, the functions from $Q\left(\bar{B}_{R}\right)$ are uniformly bounded and equicontinuous. Indeed, by using the notations (13) with $r_{1}$ replaced by $R$, we find for any $(x, y) \in \bar{B}_{R}$ and $t_{1}, t_{2} \in[0,1], t_{1}<t_{2}$ that

$$
\begin{aligned}
& \left|Q_{1}(x, y)\left(t_{2}\right)-Q_{1}(x, y)\left(t_{1}\right)\right| \leq \frac{\Psi_{R}}{\Gamma(\alpha+1)}\left(t_{2}^{\alpha}-t_{1}^{\alpha}\right)+\left(\Psi_{R} M_{11}+\Theta_{R} M_{10}\right)\left(t_{2}^{\alpha-1}-t_{1}^{\alpha-1}\right), \\
& \left|Q_{2}(x, y)\left(t_{2}\right)-Q_{2}(x, y)\left(t_{1}\right)\right| \leq \frac{\Theta_{R}}{\Gamma(\beta+1)}\left(t_{2}^{\beta}-t_{1}^{\beta}\right)+\left(\Psi_{R} M_{9}+\Theta_{R} M_{12}\right)\left(t_{2}^{\beta-1}-t_{1}^{\beta-1}\right) .
\end{aligned}
$$

Therefore we obtain

$$
\left|Q_{1}(x, y)\left(t_{2}\right)-Q_{1}(x, y)\left(t_{1}\right)\right| \rightarrow 0, \quad\left|Q_{2}(x, y)\left(t_{2}\right)-Q_{2}(x, y)\left(t_{1}\right)\right| \rightarrow 0, \quad \text { as } t_{2} \rightarrow t_{1},
$$

uniformly with respect to $(x, y) \in \bar{B}_{R}$. By Arzela-Ascoli theorem, we conclude that $Q\left(\bar{B}_{R}\right)$ is relatively compact, and then $Q$ is a compact operator. By using the Schauder fixed point theorem, we infer that operator $Q$ has at least one fixed point $(x, y)$ in $\bar{B}_{R}$, which is a solution of our problem $(S)-(B C)$.

\section{Theorem 3.6 Suppose that (J1) and}

(J6) The functions $f, g:[0,1] \times \mathbb{R}^{4} \rightarrow \mathbb{R}$ are continuous and there exist the constants $p_{i} \geq$ $0, i=0, \ldots, 4$ with at least one nonzero constant, the constants $q_{i} \geq 0, i=0, \ldots, 4$ with at least one nonzero constant, and nondecreasing functions $\xi_{i}, \eta_{i} \in C([0, \infty),[0, \infty))$ $i=1, \ldots, 4$ such that

$$
\begin{aligned}
& \left|f\left(t, u_{1}, u_{2}, u_{3}, u_{4}\right)\right| \leq p_{0}+\sum_{i=1}^{4} p_{i} \xi_{i}\left(\left|u_{i}\right|\right), \\
& \left|g\left(t, u_{1}, u_{2}, u_{3}, u_{4}\right)\right| \leq q_{0}+\sum_{i=1}^{4} q_{i} \eta_{i}\left(\left|u_{i}\right|\right),
\end{aligned}
$$

for all $t \in[0,1], u_{i} \in \mathbb{R}, i=1, \ldots, 4$, 
hold. If there exists $\Xi_{0}>0$ such that

$$
\begin{aligned}
& \left(p_{0}+p_{1} \xi_{1}\left(\Xi_{0}\right)+p_{2} \xi_{2}\left(\Xi_{0}\right)+p_{3} \xi_{3}\left(\frac{\Xi_{0}}{\Gamma\left(\theta_{1}+1\right)}\right)+p_{4} \xi_{4}\left(\frac{\Xi_{0}}{\Gamma\left(\sigma_{1}+1\right)}\right)\right)\left(M_{7}+M_{9}\right) \\
& \quad+\left(q_{0}+q_{1} \eta_{1}\left(\Xi_{0}\right)+q_{2} \eta_{2}\left(\Xi_{0}\right)+q_{3} \eta_{3}\left(\frac{\Xi_{0}}{\Gamma\left(\theta_{2}+1\right)}\right)\right. \\
& \left.\quad+q_{4} \eta_{4}\left(\frac{\Xi_{0}}{\Gamma\left(\sigma_{2}+1\right)}\right)\right)\left(M_{8}+M_{10}\right)<\Xi_{0},
\end{aligned}
$$

then problem $(S)-(B C)$ has at least one solution on $[0,1]$.

Proof We consider the set $\bar{B}_{\Xi_{0}}=\left\{(x, y) \in Y,\|(x, y)\|_{Y} \leq \Xi_{0}\right\}$, where $\Xi_{0}$ is given in the theorem. We will show that $Q: \bar{B}_{\Xi_{0}} \rightarrow \bar{B}_{\Xi_{0}}$. For $(x, y) \in \bar{B}_{\Xi_{0}}$ and $t \in[0,1]$, we obtain

$$
\begin{aligned}
& \left|Q_{1}(x, y)(t)\right| \\
& \leq\left(p_{0}+p_{1} \xi_{1}\left(\Xi_{0}\right)+p_{2} \xi_{2}\left(\Xi_{0}\right)+p_{3} \xi_{3}\left(\frac{\Xi_{0}}{\Gamma\left(\theta_{1}+1\right)}\right)+p_{4} \xi_{4}\left(\frac{\Xi_{0}}{\Gamma\left(\sigma_{1}+1\right)}\right)\right) M_{7} \\
& \quad+\left(q_{0}+q_{1} \eta_{1}\left(\Xi_{0}\right)+q_{2} \eta_{2}\left(\Xi_{0}\right)+q_{3} \eta_{3}\left(\frac{\Xi_{0}}{\Gamma\left(\theta_{2}+1\right)}\right)+q_{4} \eta_{4}\left(\frac{\Xi_{0}}{\Gamma\left(\sigma_{2}+1\right)}\right)\right) M_{10} \\
& \left|Q_{2}(x, y)(t)\right| \\
& \quad \leq\left(p_{0}+p_{1} \xi_{1}\left(\Xi_{0}\right)+p_{2} \xi_{2}\left(\Xi_{0}\right)+p_{3} \xi_{3}\left(\frac{\Xi_{0}}{\Gamma\left(\theta_{1}+1\right)}\right)+p_{4} \xi_{4}\left(\frac{\Xi_{0}}{\Gamma\left(\sigma_{1}+1\right)}\right)\right) M_{9} \\
& \quad+\left(q_{0}+q_{1} \eta_{1}\left(\Xi_{0}\right)+q_{2} \eta_{2}\left(\Xi_{0}\right)+q_{3} \eta_{3}\left(\frac{\Xi_{0}}{\Gamma\left(\theta_{2}+1\right)}\right)+q_{4} \eta_{4}\left(\frac{\Xi_{0}}{\Gamma\left(\sigma_{2}+1\right)}\right)\right) M_{8}
\end{aligned}
$$

and then, for all $(x, y) \in \bar{B}_{\Xi_{0}}$, we find

$$
\begin{aligned}
& \|Q(x, y)\|_{Y} \\
& \leq\left(p_{0}+p_{1} \xi_{1}\left(\Xi_{0}\right)+p_{2} \xi_{2}\left(\Xi_{0}\right)+p_{3} \xi_{3}\left(\frac{\Xi_{0}}{\Gamma\left(\theta_{1}+1\right)}\right)+p_{4} \xi_{4}\left(\frac{\Xi_{0}}{\Gamma\left(\sigma_{1}+1\right)}\right)\right)\left(M_{7}+M_{9}\right) \\
& \quad+\left(q_{0}+q_{1} \eta_{1}\left(\Xi_{0}\right)+q_{2} \eta_{2}\left(\Xi_{0}\right)+q_{3} \eta_{3}\left(\frac{\Xi_{0}}{\Gamma\left(\theta_{2}+1\right)}\right)\right. \\
& \left.\quad+q_{4} \eta_{4}\left(\frac{\Xi_{0}}{\Gamma\left(\sigma_{2}+1\right)}\right)\right)\left(M_{8}+M_{10}\right)<\Xi_{0} .
\end{aligned}
$$

Hence $Q\left(\bar{B}_{\Xi_{0}}\right) \subset \bar{B}_{\Xi_{0}}$. Using a similar approach as in the proof of Theorem 3.5, we can show that operator $Q$ is completely continuous.

We suppose now that there exists $(x, y) \in \partial B_{\Xi_{0}}$ such that $(x, y)=v Q(x, y)$ for some $v \in$ $(0,1)$. Arguing as above, we deduce $\|(x, y)\|_{Y} \leq\|Q(x, y)\|_{Y}<\Xi_{0}$, which is a contradiction, because $(x, y) \in \partial B_{\Xi_{0}}$. Then by using the nonlinear alternative of Leray-Schauder type, we conclude that operator $Q$ has a fixed point $(x, y) \in \bar{B}_{\Xi_{0}}$, and so problem $(S)-(B C)$ has at least one solution. 


\section{Examples}

Let $\alpha=\frac{3}{2}(n=2), \beta=\frac{7}{3}(m=3), \theta_{1}=\frac{1}{4}, \sigma_{1}=\frac{6}{5}, \theta_{2}=\frac{17}{4}, \sigma_{2}=\frac{1}{3}, p=1, q=2, \gamma_{0}=\frac{1}{6}, \gamma_{1}=\frac{3}{4}$, $\delta_{0}=\frac{8}{7}, \delta_{1}=\frac{1}{5}, \delta_{2}=\frac{1}{3}, H_{1}(t)=\left\{0, t \in\left[0, \frac{1}{2}\right) ; 3, t \in\left[\frac{1}{2}, 1\right]\right\}, K_{1}(t)=-t^{2}, t \in[0,1], K_{2}(t)=\{0, t \in$ $\left.\left[0, \frac{1}{3}\right) ; 4, t \in\left[\frac{1}{3}, 1\right]\right\}$.

We consider the system of fractional differential equations

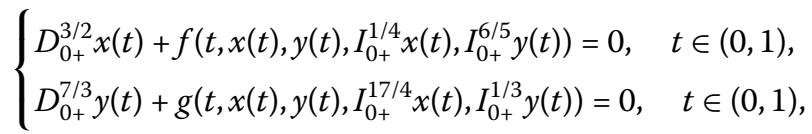

with the boundary conditions

$$
\left\{\begin{array}{l}
x(0)=0, \quad D_{0+}^{1 / 6} x(1)=3 D_{0+}^{3 / 4} y\left(\frac{1}{2}\right), \\
y(0)=y^{\prime}(0)=0, \quad D_{0+}^{8 / 7} y(1)=-2 \int_{0}^{1} t D_{0+}^{1 / 5} x(t) d t+4 D_{0+}^{1 / 3} x\left(\frac{1}{3}\right) .
\end{array}\right.
$$

We obtain $\Delta \approx-4.92715202 \neq 0$. So assumption $(J 1)$ is satisfied. In addition, we have $M_{1} \approx 2.10326265, M_{2} \approx 1.90760368, M_{3} \approx 1.02839972, M_{4} \approx 2.11984652, M_{5}=M_{1}$, $M_{6}=M_{4}, M_{7} \approx 1.81109405, M_{10} \approx 0.68108088, M_{9} \approx 0.9999811, M_{8} \approx 1.12515265$, $M_{11} \approx 1.05884127$, and $M_{12} \approx 0.76520198$.

Example 1 We consider the functions

$$
\begin{aligned}
& f\left(t, u_{1}, u_{2}, u_{3}, u_{4}\right) \\
& \quad=\frac{1}{\sqrt{9+t^{3}}}-\frac{t}{10} \arctan u_{1}+\frac{\left|u_{2}\right|}{(t+2)^{4}\left(1+\left|u_{2}\right|\right)}+\frac{1}{3(t+8)} \sin ^{2} u_{3}-\frac{t^{2}}{t+12} \cos u_{4}, \\
& g\left(t, u_{1}, u_{2}, u_{3}, u_{4}\right) \\
& \quad=\frac{3 t}{t^{2}+4}-\frac{\left|u_{1}\right|}{6\left(2+\left|u_{1}\right|\right)}+\frac{1}{15} \sin u_{2}+\frac{t}{t+24} \cos ^{2} u_{3}-\frac{1}{12} \arctan u_{4},
\end{aligned}
$$

for all $t \in[0,1], u_{i} \in \mathbb{R}, i=1, \ldots, 4$. We find the inequalities

$$
\begin{aligned}
& \mid f(t,\left.u_{1}, u_{2}, u_{3}, u_{4}\right)-f\left(t, v_{1}, v_{2}, v_{3}, v_{4}\right) \mid \\
& \leq \frac{1}{10}\left|u_{1}-v_{1}\right|+\frac{1}{16}\left|u_{2}-v_{2}\right|+\frac{1}{12}\left|u_{3}-v_{3}\right| \\
& \quad+\frac{1}{13}\left|u_{4}-v_{4}\right| \leq \frac{1}{10} \sum_{i=1}^{4}\left|u_{i}-v_{i}\right|, \\
&\left|g\left(t, u_{1}, u_{2}, u_{3}, u_{4}\right)-g\left(t, v_{1}, v_{2}, v_{3}, v_{4}\right)\right| \\
& \leq \frac{1}{12}\left|u_{1}-v_{1}\right|+\frac{1}{15}\left|u_{2}-v_{2}\right|+\frac{2}{25}\left|u_{3}-v_{3}\right| \\
& \quad+\frac{1}{12}\left|u_{4}-v_{4}\right| \leq \frac{1}{12} \sum_{i=1}^{4}\left|u_{i}-v_{i}\right|,
\end{aligned}
$$

for all $t \in[0,1], u_{i}, v_{i} \in \mathbb{R}, i=1, \ldots, 4$. So we have $L_{1}=\frac{1}{10}, L_{2}=\frac{1}{12}$ and $\Xi=L_{1} M_{5}\left(M_{7}+\right.$ $\left.M_{9}\right)+L_{2} M_{6}\left(M_{8}+M_{10}\right) \approx 0.91032<1$. Therefore assumption $(J 2)$ is satisfied, and, by Theorem 3.1, we deduce that problem $\left(S_{0}\right)-\left(B C_{0}\right)$ has at least one solution $(x(t), y(t)), t \in[0,1]$. 
Example 2 We consider the functions

$$
\begin{aligned}
& f\left(t, u_{1}, u_{2}, u_{3}, u_{4}\right)=\frac{t+2}{t^{2}+5}\left(2 \sin t+\frac{1}{5} \cos u_{1}\right)-\frac{1}{(t+5)^{2}} u_{2}-\frac{t}{6} \arctan u_{3}+\frac{1}{7} \sin u_{4} \\
& g\left(t, u_{1}, u_{2}, u_{3}, u_{4}\right)=\frac{e^{-t}}{2+t^{3}}+\frac{1}{4} \cos ^{2} u_{2}-\frac{1}{5} \sin u_{3}+\frac{1}{9} \arctan u_{4}
\end{aligned}
$$

for all $t \in[0,1], u_{i} \in \mathbb{R}, i=1, \ldots, 4$. Because we have

$$
\begin{aligned}
\left|f\left(t, u_{1}, u_{2}, u_{3}, u_{4}\right)\right| & \leq \frac{11}{10}+\frac{1}{25}\left|u_{2}\right|+\frac{1}{6}\left|u_{3}\right|+\frac{1}{7}\left|u_{4}\right|, \\
\left|g\left(t, u_{1}, u_{2}, u_{3}, u_{4}\right)\right| & \leq \frac{3}{4}+\frac{1}{5}\left|u_{3}\right|+\frac{1}{9}\left|u_{4}\right|,
\end{aligned}
$$

for all $t \in[0,1], u_{i} \in \mathbb{R}, i=1, \ldots, 4$, the assumption $(J 3)$ is satisfied with $a_{0}=\frac{11}{10}, a_{1}=0$, $a_{2}=\frac{1}{25}, a_{3}=\frac{1}{6}, a_{4}=\frac{1}{7}, b_{0}=\frac{3}{4}, b_{1}=b_{2}=0, b_{3}=\frac{1}{5}$, and $b_{4}=\frac{1}{9}$. In addition, we obtain $M_{13} \approx 0.52715168, M_{14} \approx 0.70166538$, and $\Xi_{1}=\max \left\{M_{13}, M_{14}\right\}=M_{14}<1$. Then, by Theorem 3.2, we conclude that problem $\left(S_{0}\right)-\left(B C_{0}\right)$ has at least one solution $(x(t), y(t)), t \in$ $[0,1]$.

Example 3 We consider the functions

$$
\begin{aligned}
& f\left(t, u_{1}, u_{2}, u_{3}, u_{4}\right)=-\frac{1}{5}\left|u_{2}\right|^{3 / 4}+\frac{1}{3\left(1+t^{2}\right)} \arctan \left|u_{3}\right|^{1 / 2} \\
& g\left(t, u_{1}, u_{2}, u_{3}, u_{4}\right)=\frac{e^{-t}}{1+t^{3}}-\frac{1}{3} u_{1}^{4 / 5}+\sin u_{4}^{2 / 3}
\end{aligned}
$$

for all $t \in[0,1], u_{i} \in \mathbb{R}, i=1, \ldots, 4$. Because we obtain

$$
\begin{aligned}
\left|f\left(t, u_{1}, u_{2}, u_{3}, u_{4}\right)\right| & \leq \frac{1}{5}\left|u_{2}\right|^{3 / 4}+\frac{1}{3}\left|u_{3}\right|^{1 / 2}, \\
\left|g\left(t, u_{1}, u_{2}, u_{3}, u_{4}\right)\right| & \leq 1+\frac{1}{3}\left|u_{1}\right|^{4 / 5}+\left|u_{4}\right|^{2 / 3},
\end{aligned}
$$

for all $t \in[0,1], u_{i} \in \mathbb{R}, i=1, \ldots, 4$, the assumption $(J 5)$ is satisfied with $c_{0}=c_{1}=0, c_{2}=$ $\frac{1}{5}, c_{3}=\frac{1}{3}, c_{4}=0, d_{0}=1, d_{1}=\frac{1}{3}, d_{2}=d_{3}=0, d_{4}=1, l_{2}=\frac{3}{4}, l_{3}=\frac{1}{2}, m_{1}=\frac{4}{5}$, and $m_{4}=\frac{2}{3}$. Therefore, by Theorem 3.5 , we deduce that problem $\left(S_{0}\right)-\left(B C_{0}\right)$ has at least one solution $(x(t), y(t)), t \in[0,1]$.

Example 4 We consider the functions

$$
\begin{aligned}
& f\left(t, u_{1}, u_{2}, u_{3}, u_{4}\right)=\frac{t^{3}}{25}+\frac{e^{-t} u_{1}^{4}}{20\left(1+u_{2}^{2}\right)}-\frac{t^{2} u_{4}^{1 / 3}}{10}, \\
& g\left(t, u_{1}, u_{2}, u_{3}, u_{4}\right)=\frac{(1-t)^{4}}{20}-\frac{1-t^{2}}{15} u_{2}^{2}-\frac{1}{25} u_{3}^{2 / 5},
\end{aligned}
$$

for all $t \in[0,1], u_{i} \in \mathbb{R}, i=1, \ldots, 4$. Because we have

$$
\left|f\left(t, u_{1}, u_{2}, u_{3}, u_{4}\right)\right| \leq \frac{1}{25}+\frac{1}{20}\left|u_{1}\right|^{4}+\frac{1}{10}\left|u_{4}\right|^{1 / 3}
$$




$$
\left|g\left(t, u_{1}, u_{2}, u_{3}, u_{4}\right)\right| \leq \frac{1}{20}+\frac{1}{15}\left|u_{2}\right|^{2}+\frac{1}{25}\left|u_{3}\right|^{2 / 5},
$$

for all $t \in[0,1], u_{i} \in \mathbb{R}, i=1, \ldots, 4$, the assumption (J6) is satisfied with $p_{0}=\frac{1}{25}, p_{1}=\frac{1}{20}$, $p_{2}=p_{3}=0, p_{4}=\frac{1}{10}, q_{0}=\frac{1}{20}, q_{1}=0, q_{2}=\frac{1}{15}, q_{3}=\frac{1}{25}, q_{4}=0, \xi_{1}(x)=x^{4}, \xi_{4}(x)=x^{1 / 3}$, $\eta_{2}(x)=x^{2}$, and $\eta_{3}(x)=x^{2 / 5}$ for $x \geq 0$. For $\Xi_{0}=1$, the condition (14) is satisfied because $\left(\frac{1}{25}+\frac{1}{20}+\frac{1}{10}\left(\frac{1}{\Gamma(11 / 5)}\right)^{1 / 3}\right)\left(M_{7}+M_{9}\right)+\left(\frac{1}{20}+\frac{1}{15}+\frac{1}{25}\left(\frac{1}{\Gamma(21 / 4)}\right)^{2 / 5}\right)\left(M_{8}+M_{10}\right) \approx 0.75328<1$. Then, by Theorem 3.6, we conclude that problem $\left(S_{0}\right)-\left(B C_{0}\right)$ has at least one solution $(x(t), y(t)), t \in[0,1]$.

\section{Acknowledgements}

The author thanks the referee for his/her valuable comments and suggestions.

\section{Funding}

Not applicable.

Abbreviations

Not applicable.

Availability of data and materials

Not applicable.

\section{Ethics approval and consent to participate}

Not applicable.

\section{Competing interests}

The author declares that she has no competing interests.

\section{Consent for publication}

Not applicable.

Authors' contributions

The author read and approved the final manuscript.

\section{Publisher's Note}

Springer Nature remains neutral with regard to jurisdictional claims in published maps and institutional affiliations.

Received: 20 November 2020 Accepted: 14 February 2021 Published online: 25 February 2021

\section{References}

1. Agarwal, R.P., Luca, R.: Positive solutions for a semipositone singular Riemann-Liouville fractional differential problem. Int. J. Nonlinear Sci. Numer. Simul. 20(7-8), 823-832 (2019)

2. Ahmad, B., Luca, R.: Existence of solutions for a sequential fractional integro-differential system with coupled integral boundary conditions. Chaos Solitons Fractals 104, 378-388 (2017)

3. Ahmad, B., Luca, R.: Existence of solutions for a system of fractional differential equations with coupled nonlocal boundary conditions. Fract. Calc. Appl. Anal. 21(2), 423-441 (2018)

4. Ahmad, B., Luca, R.: Existence of solutions for sequential fractional integro-differential equations and inclusions with nonlocal boundary conditions. Appl. Math. Comput. 339, 516-534 (2018)

5. Ahmad, B., Ntouyas, S.K.: Existence results for a coupled system of Caputo type sequential fractional differential equations with nonlocal integral boundary conditions. Appl. Math. Comput. 266, 615-622 (2015)

6. Aljoudi, S., Ahmad, B., Nieto, J.J., Alsaedi, A.: A coupled system of Hadamard type sequential fractional differential equations with coupled strip conditions. Chaos Solitons Fractals 91, 39-46 (2016)

7. Arafa, A.A.M., Rida, S.Z., Khalil, M.: Fractional modeling dynamics of HIV and $\mathrm{CD}^{+}{ }^{+}$T-cells during primary infection. Nonlinear Biomed. Phys. 6(1), 1-7 (2012)

8. Baleanu, D., Diethelm, K., Scalas, E., Trujillo, J.J.: Fractional Calculus Models and Numerical Methods. Series on Complexity, Nonlinearity and Chaos. World Scientific, Boston (2012)

9. Cole, K.: Electric conductance of biological systems. In: Proc. Cold Spring Harbor Symp. Quant. Biol., pp. 107-116. Springer, New York (1993)

10. Das, S.: Functional Fractional Calculus for System Identification and Controls. Springer, New York (2008)

11. Ding, Y., Ye, H.: A fractional-order differential equation model of HIV infection of CD4 ${ }^{+}$T-cells. Math. Comput. Model. 50, 386-392 (2009)

12. Djordjevic, V., Jaric, J., Fabry, B., Fredberg, J., Stamenovic, D.: Fractional derivatives embody essential features of cell rheological behavior. Ann. Biomed. Eng. 31, 692-699 (2003)

13. Ge, Z.M., Ou, C.Y.: Chaos synchronization of fractional order modified Duffing systems with parameters excited by a chaotic signal. Chaos Solitons Fractals 35, 705-717 (2008) 
14. Guo, L., Liu, L., Wu, Y.: Iterative unique positive solutions for singular $p$-Laplacian fractional differential equation system with several parameters. Nonlinear Anal., Model. Control 23(2), 182-203 (2018)

15. Henderson, J., Luca, R.: Boundary Value Problems for Systems of Differential, Difference and Fractional Equations. Positive Solutions. Elsevier, Amsterdam (2016)

16. Henderson, J., Luca, R.: Existence of positive solutions for a singular fractional boundary value problem. Nonlinear Anal., Model. Control 22(1), 99-114 (2017)

17. Henderson, J., Luca, R.: Systems of Riemann-Liouville fractional equations with multi-point boundary conditions. Appl. Math. Comput. 309, 303-323 (2017)

18. Henderson, J., Luca, R., Tudorache, A.: On a system of fractional differential equations with coupled integral boundary conditions. Fract. Calc. Appl. Anal. 18(2), 361-386 (2015)

19. Henderson, J., Luca, R., Tudorache, A.: Existence and nonexistence of positive solutions for coupled Riemann-Liouville fractional boundary value problems. Discrete Dyn. Nat. Soc. 2016, Article ID 2823971 (2016)

20. Kilbas, A.A., Srivastava, H.M., Trujillo, J.J.: Theory and Applications of Fractional Differential Equations. North-Holland Mathematics Studies, vol. 204. Elsevier, Amsterdam (2006)

21. Klafter, J., Lim, S.C., Metzler, R. (eds.): Fractional Dynamics in Physics World Scientific, Singapore (2011)

22. Krasnosel'skii, M.A.: Two remarks on the method of successive approximations. Usp. Mat. Nauk 10, 123-127 (1955)

23. Liu, L., Li, H., Liu, C., Wu, Y.: Existence and uniqueness of positive solutions for singular fractional differential systems with coupled integral boundary value problems. J. Nonlinear Sci. Appl. 10, 243-262 (2017)

24. Liu, S., Liu, J., Dai, Q., Li, H.: Uniqueness results for nonlinear fractional differential equations with infinite-point integral boundary conditions. J. Nonlinear Sci. Appl. 10, 1281-1288 (2017)

25. Luca, R.: Positive solutions for a system of Riemann-Liouville fractional differential equations with multi-point fractional boundary conditions. Bound. Value Probl. 2017, 102 (2017)

26. Luca, R., Tudorache, A.: Positive solutions to a system of semipositone fractional boundary value problems. Adv. Differ. Equ. 2014, 179, (2014)

27. Metzler, R., Klafter, J.: The random walks guide to anomalous diffusion: a fractional dynamics approach. Phys. Rep. $339,1-77(2000)$

28. Ostoja-Starzewski, M.: Towards thermoelasticity of fractal media. J. Therm. Stresses 30, 889-896 (2007)

29. Podlubny, I.: Fractional Differential Equations. Academic Press, San Diego (1999)

30. Pu, R., Zhang, X., Cui, Y., Li, P., Wang, W.: Positive solutions for singular semipositone fractional differential equation subject to multipoint boundary conditions. J. Funct. Spaces 2017, Article ID 5892616 (2017)

31. Sabatier, J., Agrawal, O.P., Machado, J.A.T. (eds.): Advances in Fractional Calculus: Theoretical Developments and Applications in Physics and Engineering Springer, Dordrecht (2007)

32. Samko, S.G., Kilbas, A.A., Marichev, O.I.: Fractional Integrals and Derivatives. Theory and Applications. Gordon \& Breach, Yverdon (1993)

33. Sokolov, I.M., Klafter, J., Blumen, A.: A fractional kinetics. Phys. Today 55, 48-54 (2002)

34. Tudorache, A., Luca, R.: Positive solutions for a system of Riemann-Liouville fractional boundary value problems with p-Laplacian operators. Adv. Differ. Equ. 2020, 292 (2020)

35. Xu, J., Wei, Z:: Positive solutions for a class of fractional boundary value problems. Nonlinear Anal., Model. Control 21, $1-17(2016)$

36. Zhang, X.: Positive solutions for a class of singular fractional differential equation with infinite-point boundary conditions. Appl. Math. Lett. 39, 22-27 (2015)

37. Zhang, X., Zhong, Q: Triple positive solutions for nonlocal fractional differential equations with singularities both on time and space variables. Appl. Math. Lett. 80, 12-19 (2018)

38. Zhou, Y.: Basic Theory of Fractional Differential Equations. World Scientific, Singapore (2014)

\section{Submit your manuscript to a SpringerOpen ${ }^{\circ}$ journal and benefit from:}

- Convenient online submission

- Rigorous peer review

- Open access: articles freely available online

- High visibility within the field

- Retaining the copyright to your article

Submit your next manuscript at $\gg$ springeropen.com 